\title{
The effect of meteorological conditions and atmospheric composition in the occurrence and development of new particle formation (NPF) events in Europe
}

\author{
Dimitrios Bousiotis ${ }^{1}$, James Brean ${ }^{1}$, Francis D. Pope ${ }^{1}$, Manuel Dall'Osto ${ }^{2}$, Xavier Querol $^{3}$, Andrés Alastuey ${ }^{3}$, \\ Noemi Perez ${ }^{3}$, Tuukka Petäjä ${ }^{4}$, Andreas Massling ${ }^{5}$, Jacob Klenø Nøjgaard ${ }^{5,6}$, Claus Nordstrøm ${ }^{5}$, \\ Giorgos Kouvarakis $^{7}$, Stergios Vratolis ${ }^{8}$, Konstantinos Eleftheriadis ${ }^{8}$, Jarkko V. Niemi ${ }^{9}$, Harri Portin ${ }^{9}$, \\ Alfred Wiedensohler ${ }^{10}$, Kay Weinhold ${ }^{10}$, Maik Merkel ${ }^{10}$, Thomas Tuch $^{10}$, and Roy M. Harrison ${ }^{1,11}$ \\ ${ }^{1}$ Division of Environmental Health and Risk Management, School of Geography, Earth and Environmental Sciences, \\ University of Birmingham, Edgbaston, Birmingham B15 2TT, UK \\ ${ }^{2}$ Institute of Marine Sciences, Passeig Marítim de la Barceloneta, 37-49, 08003, Barcelona, Spain \\ ${ }^{3}$ Institute of Environmental Assessment and Water Research (IDAEA - CSIC), 08034, Barcelona, Spain \\ ${ }^{4}$ Institute for Atmospheric and Earth System Research (INAR)/Physics, Faculty of Science University of Helsinki, Finland \\ ${ }^{5}$ Department of Environmental Science, Aarhus University, 4000 Roskilde, Denmark \\ ${ }^{6}$ The National Research Centre for the Working Environment, 2100 Copenhagen, Denmark \\ ${ }^{7}$ Environmental Chemical Processes Laboratory (ECPL), Department of Chemistry, \\ University of Crete, 70013, Heraklion, Greece \\ ${ }^{8}$ Environmental Radioactivity Laboratory, Institute of Nuclear and Radiological Science \& Technology, Energy \& Safety, \\ NCSR Demokritos, Athens, Greece \\ ${ }^{9}$ Helsinki Region Environmental Services Authority (HSY), 00066 HSY, Helsinki, Finland \\ ${ }^{10}$ Leibniz Institute for Tropospheric Research (TROPOS), Permoserstr. 15, 04318 Leipzig, Germany \\ ${ }^{11}$ Department of Environmental Sciences/Center of Excellence in Environmental Studies, \\ King Abdulaziz University, P.O. Box 80203, Jeddah, 21589, Saudi Arabia
}

Correspondence: Roy M. Harrison (r.m.harrison@bham.ac.uk)

Received: 5 June 2020 - Discussion started: 5 August 2020

Revised: 20 January 2021 - Accepted: 21 January 2021 - Published: 4 March 2021

\begin{abstract}
Although new particle formation (NPF) events have been studied extensively for some decades, the mechanisms that drive their occurrence and development are yet to be fully elucidated. Laboratory studies have done much to elucidate the molecular processes involved in nucleation, but this knowledge has yet to be conclusively linked to NPF events in the atmosphere. There is great difficulty in successful application of the results from laboratory studies to real atmospheric conditions due to the diversity of atmospheric conditions and observations found, as NPF events occur almost everywhere in the world without always following a clearly defined trend of frequency, seasonality, atmospheric conditions, or event development.
\end{abstract}

The present study seeks common features in nucleation events by applying a binned linear regression over an extensive dataset from 16 sites of various types (combined dataset of 85 years from rural and urban backgrounds as well as roadside sites) in Europe. At most sites, a clear positive relation with the frequency of NPF events is found between the solar radiation intensity (up to $R^{2}=0.98$ ), temperature (up to $R^{2}=0.98$ ), and atmospheric pressure (up to $R^{2}=0.97$ ), while relative humidity $(\mathrm{RH})$ presents a negative relation (up to $R^{2}=0.95$ ) with NPF event frequency, though exceptions were found among the sites for all the variables studied. Wind speed presents a less consistent relationship, which appears to be heavily affected by local conditions. While some meteorological variables (such as the solar radiation intensity 
and RH) appear to have a crucial effect on the occurrence and characteristics of NPF events, especially at rural sites, it appears that their role becomes less marked at higher average values.

The analysis of chemical composition data presents interesting results. Concentrations of almost all chemical compounds studied (apart from $\mathrm{O}_{3}$ ) and the condensation sink (CS) have a negative relationship with NPF event frequency, though areas with higher average concentrations of $\mathrm{SO}_{2}$ had higher NPF event frequency. Particulate organic carbon (OC), volatile organic compounds (VOCs), and particulatephase sulfate consistently had a positive relation with the growth rate of the newly formed particles. As with some meteorological variables, it appears that at increased concentrations of pollutants or the CS, their influence upon NPF frequency is reduced.

\section{Introduction}

New particle formation (NPF) events are an important source of particles in the atmosphere (Merikanto et al., 2009; Spracklen et al., 2010). These are known to have adverse effects on human health (Schwartz et al., 1996; Politis et al., 2008; Kim et al., 2015) and affect the optical and physical properties of the atmosphere (Makkonen et al., 2012; Seinfeld and Pandis, 2012). While NPF events occur almost everywhere in the world (Dall'Osto et al., 2018; Kulmala et al., 2017; O'Dowd et al., 2002; Wiedensohler et al., 2019; Chu et al., 2019; Kerminen et al., 2018), with some exceptions reported in forest (Lee et al., 2016; Pillai et al., 2013; Rizzo et al., 2010) and high-elevation sites (Bae et al., 2010; Hallar et al., 2016), great diversity is found in the atmospheric conditions within which they take place. The many studies conducted have included many different types of locations (urban, traffic, regional background) around the world, and differences were found in both the seasonality and intensity of NPF events. This variability may be related to the mix of conditions that are specific to each location, which obscures the general understanding of the conditions that are favourable for the occurrence of NPF events (Berland et al., 2017; Bousiotis et al., 2020). For example, solar radiation is considered one of the most important factors in the occurrence of NPF events (Kulmala and Kerminen, 2008; Kürten et al., 2016; Pikridas et al., 2015; Salma et al., 2011), as it drives the photochemical reactions leading to the formation of sulfuric acid (Petäjä et al., 2009; Cheung et al., 2013), which is frequently the main component of the formation and growth of the initial clusters (Iida et al., 2008; Stolzenburg et al., 2020; Weber et al., 1995). Nevertheless, in many cases NPF events do not occur in the seasons with the highest insolation (Park et al., 2015; Vratolis et al., 2019). Similarly, uncertainty exists over the effect of temperature (Yli-Juuti et al., 2020; Stolzenburg et al., 2018). Higher temperatures are considered favourable for the growth of newly formed particles as increased concentrations of both biogenic volatile organic compounds (BVOCs) and anthropogenic volatile organic compounds (AVOCs) (Yamada, 2013; Paasonen et al., 2013) as well as their oxidation products (Ehn et al., 2014) support growth of the particles. On the other hand, the negative effect of increased temperature upon the stability of molecular clusters should not be overlooked (Kürten et al, 2018; Zhang et al., 2012). The former factor appears frequently be dominant, as higher growth rates are found in most cases in the local summer (Nieminen et al., 2018), although the actual importance of VOCs in the occurrence of NPF events is still not fully elucidated, with oxidation mechanisms still under intense research (Tröstl et al., 2016; Wang et al., 2020). The effect of other meteorological variables is even more complex, with studies presenting mixed results on the effect of the wind speed and atmospheric pressure. Extreme values of those variables may be favourable for the occurrence of NPF events, as they are associated with increased mixing in the atmosphere but at the same time suppress nucleation due to increased dilution of precursors (Brines et al., 2015; Rimnácová et al., 2011; Shen et al., 2018; Siakavaras et al., 2016) or favour it due to a reduced condensation sink (CS).

The effect of atmospheric composition on NPF events is also a puzzle of mixed results. While the negative effect of increased CS on the occurrence of the events is widely accepted (Kalkavouras et al., 2017; Kerminen et al., 2004; Wehner et al., 2007), cases are found when NPF events occur on days with higher CS compared to average conditions (Größ et al., 2018; Kulmala et al., 2005). Sulfur dioxide $\left(\mathrm{SO}_{2}\right)$, which is one of the most important contributors to many NPF pathways, in most studies was found at lower concentrations on NPF event days compared to average conditions (Alam et al., 2003; Bousiotis et al., 2019), although there are studies that have reported the opposite (Woo et al., 2001; Charron et al., 2008). Additionally, in a combined study of NPF events in China, events were found to be more probable under sulfur-rich rather than sulfur-poor conditions (Jayaratne et al., 2017). The case with BVOCs and AVOCs is similar, which present great variability depending on the area studied (Dai et al., 2017), and their contribution to the growth of the particles is not fully understood yet. Until recently, it was considered unlikely for NPF events, as they are considered in the present study (deriving from secondary formation not associated with traffic-related processes such as dilution of engine exhaust), to occur within the complex urban environment due to the increased presence of compounds mainly associated with combustion processes, which would suppress the survival of the newly formed particles within this type of environment (Kulmala et al., 2017). Despite this, NPF events were found to occur within even the most polluted areas and sometimes with high formation and growth rates (Bousiotis et al., 2019; Yao et al., 2018). 
It is evident that while general knowledge of the role of the meteorological and atmospheric variables has been achieved, there is great uncertainty over the extent and variability of their effect (and for some of them even the direction of an effect) in the mechanisms of NPF in real atmospheric conditions, especially in the more complex urban environment (Harrison, 2017). The present study, using an extensive dataset from 16 sites in six European countries, attempts to elucidate the effect of several meteorological and atmospheric variables not only in general, but also depending on the geographical region or type of environment. While studies with multiple sites have been reported in the past (Dall'Osto et al., 2018; Kulmala et al., 2005; Rivas et al., 2020), to the authors' knowledge this is the first study that focuses directly on the effect of these variables upon the frequency of NPF events as well as the formation and growth rates of newly formed particles in real atmospheric conditions.

\section{Data and methods}

\subsection{Site description and data availability}

The present study uses a total of more than 85 years of hourly data from 16 sites from six countries in Europe with various land usage and climates. It was considered very important that at least a rural and an urban site would be available from each country to study the differences between the different land usage effects on NPF events throughout Europe. The sites were chosen to cover the greatest possible extent of the European continent, with sites from northern, central, and southern Europe, as well as from western and eastern Europe. The sites are located in the UK (London and Harwell), Denmark (greater Copenhagen area), Germany (greater Leipzig area), Finland (Helsinki and Hyytiälä), Spain (Barcelona and Montseny - a site in a mountainous area), and Greece (Athens and Finokalia). Unfortunately, not all sites had available data for all the variables studied, which to an extent may bias some of the results. An extended analysis of the typical and NPF event conditions, seasonal variations, and trends at these sites for the same period is found in other studies (Bousiotis et al., 2019, 2020). A list of the available data and a brief description for each site are found in Table 1 (for ease of reading the sites are named by the country of the site followed by the last two letters, which refer to the type of site: $\mathrm{RU}$ is for rural-regional background, UB is for urban background, and RO is for roadside site), while a map of the sites is found in Fig. 1. For all the sites, the data used in the present study are of either $1 \mathrm{~h}$ resolution or less. Data with coarser resolution were omitted for reliability.

Most of the data used in this analysis were also published in previous studies. The data from the UK were published in Bousiotis et al. (2019, 2020), while some were also published in Beddows et al. (2015), Beddows and Harrison (2019). The

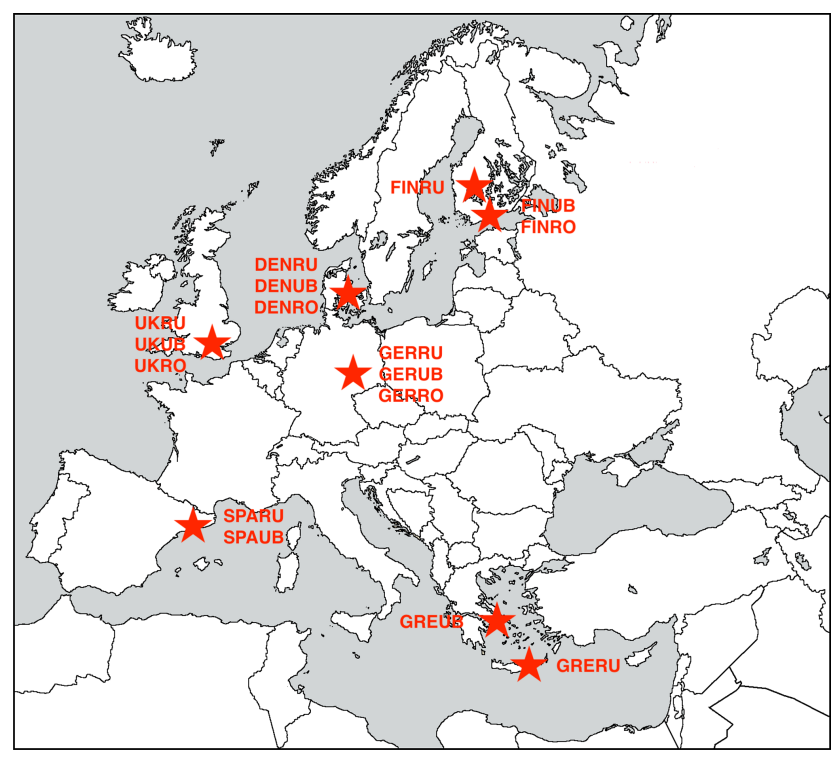

Figure 1. Map of the sites in the present study.

data for the German sites and some of the data from the UK, Denmark, and Finland were also published in von Bismarck et al. (2013, 2014, 2015). Some of the measurements for the Spanish sites were used in Carnerero et al. (2019) and Brines et al. (2015). The data for the Greek rural background site were published in Kalivitis et al. (2019). Finally, the data for the Greek urban background site were extracted from the European database (EBAS - http://ebas.nilu.no, last access: 6 November 2018) and to the authors' knowledge have not been used in previous studies. Additional data for some of the sites were provided from their respective operators and were also not used in the past.

\subsection{Methods}

\subsubsection{NPF events selection}

NPF events were selected using the method proposed by Dal Maso et al. (2005). An NPF event is identified by the appearance of a new mode or particles in the nucleation mode (smaller than $20 \mathrm{~nm}$ in diameter), which prevails for some hours and shows signs of growth. The events can then be classified into classes I and II according to the level of certainty, while class I events can be further classified to Ia and Ib. Events having both a clear formation of a new mode of particles in the smallest size bins available (thus excluding possible advected events) and a distinct and persistent growth of the new mode of particles for at least $3 \mathrm{~h}$ were classified as Ia, while Ib consists of rather clear events that fail by at least one of the criteria set. Additionally, for the roadside sites, a formation of particles in the nucleation mode accompanied by a significant increase in the concentrations of pollutants was not considered an NPF event, as it may be associated with mechanisms other than secondary formation. 
Table 1. Location and data availability of the sites.

\begin{tabular}{|c|c|c|c|c|c|}
\hline Site & Location & Available data & $\begin{array}{l}\text { Meteorological data lo- } \\
\text { cation }\end{array}$ & Data availability & Reference \\
\hline UKRU & $\begin{array}{l}\text { Harwell Science Centre, Ox- } \\
\text { ford, } 80 \mathrm{~km} \mathrm{~W} \text { of London, UK } \\
\left(51^{\circ} 34^{\prime} 15^{\prime \prime} \mathrm{N} ; 1^{\circ} 19^{\prime} 31^{\prime \prime} \mathrm{W}\right)\end{array}$ & $\begin{array}{l}\text { SMPS }(16.6-604 \mathrm{~nm}, \quad 76.5 \% \\
\text { availability), } \\
\mathrm{NO}_{x}, \mathrm{SO}_{2}, \mathrm{O}_{3}, \mathrm{OC}, \mathrm{SO}_{4}^{2-} \text {, } \\
\text { gaseous ammonia }\end{array}$ & On site & 2009-2015 & Charron et al. (2013) \\
\hline UKUB & $\begin{array}{l}\text { North Kensington, } 4 \mathrm{~km} \mathrm{~W} \text { of } \\
\text { London city centre, } \mathrm{UK} \\
\left(51^{\circ} 31^{\prime} 15^{\prime \prime} \mathrm{N} ; 0^{\circ} 12^{\prime} 48^{\prime \prime} \mathrm{W}\right)\end{array}$ & $\begin{array}{l}\text { SMPS }(16.6-604 \mathrm{~nm}, 83.3 \% \\
\text { availability), } \\
\mathrm{NO}_{x}, \mathrm{SO}_{2}, \mathrm{O}_{3}, \mathrm{OC}, \mathrm{SO}_{4}^{2-}\end{array}$ & Heathrow airport & $2009-2015$ & Bigi and Harrison (2010) \\
\hline UKRO & $\begin{array}{l}\text { Marylebone Road, London, UK } \\
\left(51^{\circ} 31^{\prime} 21^{\prime \prime} \mathrm{N} ; 0^{\circ} 9^{\prime} 16^{\prime \prime} \mathrm{W}\right)\end{array}$ & $\begin{array}{l}\text { SMPS }(16.6-604 \mathrm{~nm}, \quad 74.3 \% \\
\text { availability), } \\
\mathrm{NO}_{x}, \mathrm{SO}_{2}, \mathrm{O}_{3}, \mathrm{OC}, \mathrm{SO}_{4}^{2-}\end{array}$ & Heathrow airport & 2009-2015 & Charron and Harrison (2003) \\
\hline DENRU & $\begin{array}{l}\text { Lille Valby, } 25 \mathrm{~km} \mathrm{~W} \text { of } \\
\text { Copenhagen }\left(55^{\circ} 41^{\prime} 41^{\prime \prime} \mathrm{N} \text {; }\right. \\
\left.12^{\circ} 7^{\prime} 7^{\prime \prime} \mathrm{E}\right)(2008-\mathrm{Jun} 2010) \\
\text { Risø, } 7 \mathrm{~km} \text { north of Lille Valby } \\
\left(55^{\circ} 38^{\prime} 40^{\prime \prime} \mathrm{N} ; 12^{\circ} 5^{\prime} 19^{\prime \prime} \mathrm{E}\right) \\
(\mathrm{Jul} 2010-2017)\end{array}$ & $\begin{array}{l}\text { DMPS and } \mathrm{CPC}(5.8-700 \mathrm{~nm} \text {, } \\
68.3 \% \text { availability }) \\
\mathrm{NO}_{x}, \mathrm{SO}_{2}, \mathrm{O}_{3}, \mathrm{OC}, \mathrm{SO}_{4}^{2-}\end{array}$ & $\begin{array}{l}\text { H.C. Ørsted - Institute } \\
\text { station }\end{array}$ & 2008-2017 & Ketzel et al. (2004) \\
\hline DENRO & $\begin{array}{l}\text { H.C. Andersens Boulevard, } \\
\text { Copenhagen, } \quad \text { Denmark } \\
\left(55^{\circ} 40^{\prime} 28^{\prime \prime} \mathrm{N} ; 12^{\circ} 34^{\prime} 16^{\prime \prime} \mathrm{E}\right)\end{array}$ & $\begin{array}{l}\text { DMPS and } \mathrm{CPC}(5.8-700 \mathrm{~nm} \text {, } \\
65.7 \% \text { availability), } \\
\mathrm{NO}_{x}, \mathrm{SO}_{2}, \mathrm{O}_{3}, \mathrm{OC}, \mathrm{SO}_{4}^{2-}\end{array}$ & $\begin{array}{l}\text { H.C. Ørsted - Institute } \\
\text { station }\end{array}$ & 2008-2017 & Wang et al. (2010) \\
\hline GERRU & $\begin{array}{l}\text { Melpitz, } 40 \mathrm{~km} \mathrm{NE} \text { of Leipzig, } \\
\text { Germany }\left(51^{\circ} 31^{\prime} 31.85^{\prime \prime} \mathrm{N}\right. \\
\left.12^{\circ} 26^{\prime} 40.30^{\prime \prime} \mathrm{E}\right)\end{array}$ & $\begin{array}{l}\text { TDMPS with } \mathrm{CPC}(4.8- \\
800 \mathrm{~nm}, 87.2 \% \text { availability }) \\
\mathrm{OC}, \mathrm{SO}_{4}^{2-}\end{array}$ & On site & $2008-2011$ & Birmili et al. (2016) \\
\hline GERUB & $\begin{array}{l}\text { Tropos, } 3 \mathrm{~km} \mathrm{NE} \text { from the city } \\
\text { centre of Leipzig, } \\
\text { Germany }\left(51^{\circ} 21^{\prime} 9.1^{\prime \prime} \mathrm{N} \text {; }\right. \\
\left.12^{\circ} 26^{\prime} 5.1^{\prime \prime} \mathrm{E}\right)\end{array}$ & $\begin{array}{l}\text { TDMPS with CPC }(3-800 \mathrm{~nm} \text {, } \\
90.4 \% \text { availability })\end{array}$ & On site & 2008-2011 & Birmili et al. (2016) \\
\hline FINUB & $\begin{array}{l}\text { Kumpula Campus } 4 \mathrm{~km} \mathrm{~N} \\
\text { of the city centre, Helsinki, } \\
\text { Finland }\left(60^{\circ} 12^{\prime} 10.52^{\prime \prime} \mathrm{N} \text {; }\right. \\
\left.24^{\circ} 57^{\prime} 40.20^{\prime \prime} \mathrm{E}\right)\end{array}$ & $\begin{array}{l}\text { TDMPS with CPC }(3.4- \\
1000 \mathrm{~nm}, 99.7 \% \text { availability })\end{array}$ & On site & $\begin{array}{l}2008-2011 \& \\
2015-2018\end{array}$ & Järvi et al. (2009) \\
\hline FINRO & $\begin{array}{l}\text { Mäkelänkatu street, Helsinki, } \\
\text { Finland }\left(60^{\circ} 11^{\prime} 47.57^{\prime \prime} \mathrm{N} \text {; }\right. \\
\left.24^{\circ} 57^{\prime} 6.01^{\prime \prime} \mathrm{E}\right)\end{array}$ & $\begin{array}{l}\text { DMPS }(6-800 \mathrm{~nm}, \quad 90.0 \% \\
\text { availability), } \\
\mathrm{NO}_{x}, \mathrm{O}_{3}\end{array}$ & $\begin{array}{l}\text { Pasila station and on } \\
\text { site }\end{array}$ & $2015-2018$ & Hietikko et al. (2018) \\
\hline SPARU & $\begin{array}{l}\text { Montseny, } \quad 50 \mathrm{~km} \text { NNE } \\
\text { from Barcelona, Spain } \\
\left(41^{\circ} 46^{\prime} 45^{\prime \prime} \mathrm{N} ; 2^{\circ} 21^{\prime} 29^{\prime \prime} \mathrm{E}\right)\end{array}$ & $\begin{array}{l}\text { SMPS }(9-856 \mathrm{~nm}, 53.7 \% \text { avail- } \\
\text { ability), } \\
\mathrm{NO}_{2}, \mathrm{SO}_{2}, \mathrm{O}_{3}\end{array}$ & On site & $2012-2015$ & Dall'Osto et al. (2013) \\
\hline SPAUB & $\begin{array}{l}\text { Palau Reial, Barcelona, } \\
\text { Spain }\left(41^{\circ} 23^{\prime} 14^{\prime \prime} \mathrm{N} \text {; }\right. \\
\left.\text { 2ldegree6 } 56^{\prime \prime} \mathrm{E}\right)\end{array}$ & $\begin{array}{l}\mathrm{SMPS}(11-359 \mathrm{~nm}, \quad 88.1 \% \\
\text { availability), } \\
\mathrm{NO}_{2}, \mathrm{SO}_{2}, \mathrm{O}_{3}\end{array}$ & On site & 2012-2015 & Dall'Osto et al. (2012) \\
\hline GRERU & $\begin{array}{l}\text { Finokalia, } 70 \mathrm{~km} \mathrm{E} \text { of Her- } \\
\text { aklion, Greece }\left(35^{\circ} 20^{\prime} 16.8^{\prime \prime} \mathrm{N} \text {; }\right. \\
\left.25^{\circ} 40^{\prime} 8.4^{\prime \prime} \mathrm{E}\right)\end{array}$ & $\begin{array}{l}\text { SMPS }(8.77-849 \mathrm{~nm}, 85.0 \% \\
\text { availability), } \\
\mathrm{NO}_{2}, \mathrm{O}_{3}, \mathrm{OC}\end{array}$ & On site & $2012-2018$ & Kalkavouras et al. (2017) \\
\hline GREUB & $\begin{array}{l}\text { "Demokritos", } 12 \mathrm{~km} \mathrm{NE} \text { from } \\
\text { the city centre, Athens, Greece } \\
\left(37^{\circ} 59^{\prime} 41.96^{\prime \prime} \mathrm{N} \text {; }\right. \\
\left.23^{\circ} 48^{\prime} 57.56^{\prime \prime} \mathrm{E}\right)\end{array}$ & $\begin{array}{l}\text { SMPS }(10-550 \mathrm{~nm}, \quad 88.0 \% \\
\text { availability) }\end{array}$ & On site & 2015-2018 & Mølgaard et al. (2013) \\
\hline
\end{tabular}


In the present study, only events of class Ia were considered, with the additional criterion of at least $1 \mathrm{~nm} \mathrm{~h}^{-1}$ growth for at least $3 \mathrm{~h}$. As the available SMPS datasets for the sites in the UK are for particles with a diameter greater than $16 \mathrm{~nm}$, additional criteria were set to ensure the correct extraction of NPF events, including variations of the particle number concentrations from a condensation particle counter $(\mathrm{CPC}$ - measuring particles with diameters from $7 \mathrm{~nm}$ ) and of the concentrations of gaseous pollutants and aerosol constituents (please refer to the Methods section in Bousiotis et al., 2019).

\subsubsection{Calculation of condensation sink, growth rate, formation rate, and NPF event frequency}

The condensation sink (CS) is calculated according to the method proposed by Kulmala et al. (2001) as

$\mathrm{CS}=4 \pi D_{\text {vap }} \sum \beta_{M} r N$,

where $r$ and $N$ are the radius and number concentration of the particles, respectively, and $D_{\text {vap }}$ is the diffusion coefficient calculated as (Poling et al., 2001)

$$
D_{\text {vap }}=0.00143 \cdot T^{1.75} \frac{\sqrt{M_{\text {air }}^{-1}+M_{\text {vap }}^{-1}}}{P\left(D_{x, \text { air }}^{\frac{1}{3}} D_{x, \text { vap }}^{\frac{1}{3}}\right)^{2}}
$$

for $T=293 \mathrm{~K}$ and $P=1013.25 \mathrm{mbar}$. $M$ and $D_{x}$ are the molar mass and diffusion volume for air and sulfuric acid. $\beta_{M}$ is the Fuchs correction factor calculated as (Fuchs and Sutugin, 1971)

$\beta_{M}=\frac{1+K n}{1+\left(\frac{4}{3 a}+0.377\right) K n+\frac{4}{3 a} K n^{2}}$,

where $K n$ is the Knudsen number calculated as $K n=$ $2 \lambda_{\mathrm{m}} / d_{\mathrm{p}}$, where $\lambda_{\mathrm{m}}$ is the mean free path of the gas. It should be noted that due to the lack of sufficient chemical composition data for a number of sites, the CS calculated is not corrected for hygroscopic growth. As a result, the values for CS and the results associated with it presented in this work may be biased between the sites studied due to the large differences in the conditions between them.

Growth rate (GR) is calculated as (Kulmala et al., 2012)

$\mathrm{GR}=\frac{D_{P_{2}}-D_{P_{1}}}{t_{2}-t_{1}}$

for the size range between the minimum available particle diameter up to $30 \mathrm{~nm}(50 \mathrm{~nm}$ for the UK sites due to the higher minimum particle size available). The time window used for the calculation of the growth rate was from the start of the event until (a) growth stopped, (b) GMD reached the upper limit set, or (c) the day ended.

The formation rate $J$ was calculated using the method proposed by Kulmala et al. (2012) as

$J_{d_{\mathrm{p}}}=\frac{\mathrm{d} N_{d_{\mathrm{p}}}}{\mathrm{d} t}+\operatorname{CoagS}_{d_{\mathrm{p}}} \times N_{d_{\mathrm{p}}}+\frac{\mathrm{GR}}{\Delta d_{\mathrm{p}}} \times N_{d_{\mathrm{p}}}+S_{\text {losses }}$, where CoagS ${d_{\mathrm{p}}}_{\mathrm{p}}$ is the coagulation rate of particles of diameter $d_{\mathrm{p}}$, calculated as (Kerminen et al., 2001)

$$
\begin{aligned}
\operatorname{CoagS}_{d_{\mathrm{p}}} & =\int K\left(d_{\mathrm{p}}, d_{\mathrm{p}}^{\prime}\right) n\left(d_{\mathrm{p}}^{\prime}\right) \mathrm{d} d_{\mathrm{p}}^{\prime} \\
& \cong \sum_{d_{\mathrm{p}}^{\prime}=d_{\mathrm{p}}}^{d_{\mathrm{p}}^{\prime}=\max } K\left(d_{\mathrm{p}}, d_{\mathrm{p}}^{\prime}\right) N_{d_{\mathrm{p}}} .
\end{aligned}
$$

$K\left(d_{\mathrm{p}}, d_{\mathrm{p}}^{\prime}\right)$ is the coagulation coefficient of particles with diameters $d_{\mathrm{p}}$ and $d_{\mathrm{p}}^{\prime}$, while $S_{\text {losses }}$ accounts for additional loss terms (i.e. chamber wall losses), which are not applicable in the present study. For the present study, the formation rate of particles with a diameter of $10 \mathrm{~nm}$ was calculated for uniformity ( $16 \mathrm{~nm}$ for the UK sites), though most sites had data for particle sizes below $10 \mathrm{~nm}$.

The NPF frequency was calculated as the number of NPF event days divided by the number of days with available data in the given group (full dataset or temporal and variable ranges, etc.). The results presented in this study were normalised according to the data availability as

$\mathrm{NPF}_{\text {frequency }}=\frac{N_{\mathrm{NPF} \text { event days for group of days } X}}{N_{\text {days with available data for group of days } X}}$.

Finally, the $p$ values reported in the analysis derive from the ANOVA one-way test. As the normality of the variables is required for such an analysis, the Shapiro-Wilk test was used to assess the normality, and the vast majority of the variables were found to have $p>0.05$ and were thus considered normal. This is probably due to the removal of the extreme values (as mentioned in Sect. 2.2.3, for the calculations $90 \%$ of each dataset was kept by removing the extremely high and/or low values and the possible outliers included in them). While this was not done to promote the normality of the populations but to reduce the bias from extreme values, it indirectly assisted in making the distributions normal. For the few remaining (e.g. the growth rates associated with $\mathrm{SO}_{2}$ concentrations for UKRO) for which normality was not present, the square root of the values of the variable were considered to achieve normality and proceed to the ANOVA test.

\subsubsection{Calculation of the gradient and intercept for the variables used}

Due to the large datasets available and the large spread of the values, a direct comparison between a given variable and any of the characteristics associated with NPF events (NPF frequency, growth rate, and formation rate) always provided results with low statistical significance. As a result, an alternative method which can provide a reliable result without the dispersion of the large datasets was used in the present study to investigate the relationships between the variables considered to be associated with NPF events. For this, a timeframe which is more directly associated with the NPF events typically observed at the mid-latitudes was chosen. For NPF fre- 
quency and GR the timeframe between 05:00 and 17:00 local time (LT) was chosen, which is considered the time when the vast majority of NPF events take place and further develop with the growth of the particles. For the formation rate a smaller timeframe was chosen of 09:00 to 15:00 LT, which is $\pm 3 \mathrm{~h}$ from the time of the maximum formation rate found for almost all sites (12:00 LT). This was done to exclude as far as possible the effect of the morning rush at the roadside sites and to include only the time window when the formation rate is most relevant to NPF events (negative values that are more probable outside this timeframe and are not associated with the formation of the particles would bias the results).

For the CS the timeframe 05:00 to 10:00 LT was chosen. This was done to avoid including the direct effect of the NPF events (the contribution of newly formed particles to CS) and to provide results for conditions which either promote or suppress the characteristics studied, which specifically for the CS are more important before the start of the events. The extreme values (very high or very low) which bias the results only carrying a very small piece (forming bins of very small size) of information were then removed, though $90 \%$ of the available data was used for all the variables. The remaining data were separated into smaller bins, and a minimum of 10 bins was required for each variable (for example, if the difference between the minimum and the maximum $\mathrm{RH}$ is $70 \%$, then 14 bins each with a range of $5 \%$ were formed). The variables of interest were then averaged for each bin and plotted, and a linear relation was considered for each one of them. While it is evident that not all relationships are linear, the specific type was chosen in the present analysis for all the variables studied. This was done because the aim was to elucidate the general positive or negative effect of the variables studied. Furthermore, the effect of many variables appears to vary between sites with large differences (either geographical or type of land use), and the choice of a single method to describe these relationships ensures the uniformity of the results, as it appears to better describe them in most cases.

The gradient of these linear relations $\left(a_{\mathrm{N}}, a_{\mathrm{G}}\right.$, and $a_{J}$ for NPF frequency, growth rate, and formation rate $J_{10}$, respectively) found in this analysis should be used with great caution, as apart from the atmospheric conditions (local and meteorological as well as atmospheric composition) it is also affected by the variable in question (e.g. a higher NPF frequency will provide a larger gradient), resulting in the same trend for all the atmospheric variables tested; the sites with higher values of these variables (NPF frequency and formation rate) always had greater gradient values and vice versa. In order to remove the effect of the variable in question (NPF frequency or formation rate - the growth rate will provide an unreliable result as it is calculated in a different range for each site due to the lower available size of particles), the gradients were normalised by dividing them by their respective variable (e.g. divide the gradient of the NPF frequency by the NPF frequency), providing a new normalised slope ( $a_{\mathrm{N}}^{*}$ for NPF frequency or $a_{J}^{*}$ for the formation rate) that will have no significance other than its absolute value, which can be used for direct comparisons:

$a_{\mathrm{N}}^{*}=\frac{a_{\mathrm{N}}}{\mathrm{NPF} \%}$,

where $a_{\mathrm{N}}$ is the gradient of the relation between the given variable and NPF frequency (NPF \%),

$a_{J}^{*}=\frac{a_{J}}{J_{10}}$,

where $a_{J}$ is the gradient of the relation between the given variable and the formation rate of $10 \mathrm{~nm}$ particles $J_{10}\left(J_{16}\right.$ for the UK sites).

\section{Results}

In this study NPF events are generally observed as particles grow from a smaller size (typically $3-16 \mathrm{~nm}$ depending on the size detection limit of instruments used) to $30 \mathrm{~nm}$ or larger. They therefore reflect the result both of nucleation, which creates new particles of 1-2 nm (not detected with the instruments used in this study), and growth to larger sizes. In analysing NPF events, we therefore consider three diagnostic features.

- The first is the frequency of events occurring (i.e. days with an event divided by total days with relevant data, depending on the variable and range studied). As only class Ia events were considered, the frequency of the events calculated should be lower than the expected one if all types of events were included. This could result in values up to one-third of those anticipated if all types of events were considered. For the extent of this variation please refer to Bousiotis et al. $(2019,2020)$ in which there is an extended analysis of the NPF events for each site, including the special cases of NPF events that do not comply with the criteria set for class Ia.

- The second is the rate of particle formation at a given size ( $J_{10}$ in this case), which was found to have unclear seasonal trends among the sites and was higher for urban sites compared to rural sites in most cases (Bousiotis, 2019, 2020)

- The third is the growth rate of particles from the lower measurement limit to $30 \mathrm{~nm}$ (or $50 \mathrm{~nm}$ for the UK sites), which was found to be greater during summer months for most of the sites also studied in the aforementioned works.

From the analysis of the extended dataset a total of 1952 NPF events were extracted and studied. The NPF frequency, growth, and formation rate for each site is found in Table 2. The seasonal variation of NPF events is found in Fig. S14. 
Table 2. Frequency (and number), growth, and formation rate of class Ia NPF events.

\begin{tabular}{lrrr}
\hline Site & $\begin{array}{r}\text { Frequency of } \\
\text { NPF events }(\%)\end{array}$ & $\begin{array}{r}\text { GR } \\
\left(\mathrm{nm} \mathrm{h}^{-1}\right)\end{array}$ & $\begin{array}{r}J_{10} \\
\left(\mathrm{~N} \mathrm{~cm}^{-3} \mathrm{~s}^{-1}\right)\end{array}$ \\
\hline UKRU & $7.0(160)$ & $3.4^{\mathrm{a}}$ & $8.69 \times 10^{-3} \mathrm{~b}$ \\
UKUB & $7.0(156)$ & $4.2^{\mathrm{a}}$ & $1.42 \times 10^{-2} \mathrm{~b}$ \\
UKRO & $6.1(120)$ & $5.5^{\mathrm{a}}$ & $3.75 \times 10^{-2} \mathrm{~b}$ \\
DENRU & $7.9(176)$ & 3.19 & $2.57 \times 10^{-2}$ \\
DENUB & $5.8(116)$ & 3.19 & $2.40 \times 10^{-2}$ \\
DENRO & $5.4(117)$ & 4.45 & $8.07 \times 10^{-2}$ \\
GERRU & $17.1(164)$ & 4.34 & $9.18 \times 10^{-2}$ \\
GERUB & $17.5(169)$ & 4.24 & $1.02 \times 10^{-1}$ \\
GERRO & $9.0(62)$ & 5.17 & $1.38 \times 10^{-1}$ \\
FINRU & $8.7(190)$ & 2.91 & $1.19 \times 10^{-2}$ \\
FINUB & $5.0(110)$ & 2.87 & $2.49 \times 10^{-2}$ \\
FINRO & $5.1(49)$ & 3.74 & $6.94 \times 10^{-2}$ \\
SPARU & $12(68)$ & 3.87 & $1.54 \times 10^{-2}$ \\
SPAUB & $13.1(97)$ & 3.71 & $2.12 \times 10^{-2}$ \\
GRERU & $6.5(116)$ & 3.68 & $4.90 \times 10^{-3}$ \\
GREUB & $8.5(82)$ & 3.4 & $4.41 \times 10^{-2}$ \\
\hline
\end{tabular}

${ }^{\text {a }}$ GR up to $50 \mathrm{~nm}$ calculated; ${ }^{\mathrm{b}} J_{16}$ calculated.

\subsection{Meteorological conditions}

The gradients, coefficients of determination $\left(R^{2}-\right.$ the relationships found are characterised as weak for $R^{2}<0.50$, strong for $0.50<R^{2}<0.75$, and very strong for $R^{2}>0.75$ ), and the $p$ values from the analysis of the meteorological variables, as well as the average conditions of these variables, are found in Table 3. The results for each site and variable are found in Figs. S1-S5.

\subsubsection{Solar radiation intensity}

As mentioned earlier, solar radiation intensity is considered to be one of the most important variables in NPF occurrence, as it contributes to the production of $\mathrm{H}_{2} \mathrm{SO}_{4}$, which is a main component of the initial clusters and participates in the early growth of the newly formed particles. Hidy (1994) reported up to 6 times higher $\mathrm{SO}_{2}$ oxidation rates into $\mathrm{H}_{2} \mathrm{SO}_{4}$ in typical summer conditions compared to winter. For almost all sites this relation is confirmed with very strong correlations $\left(R^{2}>0.75\right)$ between the intensity of solar radiation and the frequency of NPF events. The relationship between the solar radiation and NPF frequency was positive at all sites, and only three sites (FINUB, SPARU, and GREUB) presented weak correlations $\left(R^{2}<0.40\right)$. Weaker correlations were found for the southern European sites, which might be associated with the higher averages for solar radiation intensity or the interference of other processes (such as coinciding with increased CS by recirculation of air masses; Carnerero et al., 2019), possibly making it less of an important factor for these areas.

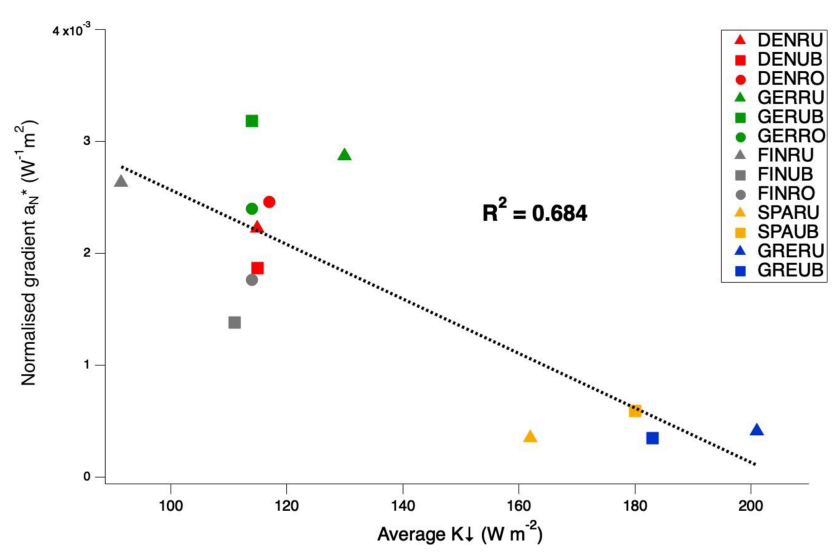

Figure 2. Relation of average downward incoming solar radiation $(K \downarrow)$ and normalised gradients $a_{\mathrm{N}}^{*}$.

The relationship of solar radiation with the growth rate was weaker in all cases and did not present a clear trend. Only some rural background sites (GERRU, FINRU, and GRERU) presented a strong correlation $\left(R^{2}>0.50\right)$. The relationship found in most cases was positive apart from two roadside sites (GERRO and UKRO) and two urban background sites (GREUB and UKUB), though due to the low $R^{2}(<0.10)$ these results cannot be considered with confidence. It seems that the solar radiation intensity is probably a more important factor at background sites rather than at roadside sites, where local conditions (such as local emissions) are possibly more important (Olin et al., 2020). Finally, the formation rate has a positive relationship with the solar radiation intensity, with relatively strong correlations in most areas $\left(R^{2}>0.50\right)$. The correlations were stronger at the rural background sites compared to the roadside sites, which further underlines the increased importance of this factor at this type of site. A negative relationship between the solar radiation intensity and the formation rate was found at the GRERU site, but the $R^{2}$ is very low $\left(R^{2}=0.05\right)$.

Plotting the normalised gradients for NPF event frequency $a_{\mathrm{N}}^{*}$ with the average solar radiation intensity at each site (Fig. 2), a negative relationship is found $\left(R^{2}=0.62\right)$, with the southern areas (those with higher average solar intensity) having smaller $a_{\mathrm{N}}^{*}$ compared to those at higher latitudes (and thus with lower average solar radiation). This may indicate that while solar radiation is a deciding factor in the occurrence of an NPF event, when in greater intensity its role becomes relatively less important, a finding that was also implied by Wonaschütz et al. (2015). Additionally, the $a_{J}^{*}$ was found to be higher at all rural sites compared to their respective roadside sites (and urban background sites for all but the Greek and German ones), making it a more important factor at this type of site (Fig. 3). 
Table 3. Normalised gradients (non-normalised for growth rate), $R^{2}$, and $p$ values ( - for values $>0.05$ ) for the relation between meteorological conditions and NPF event variables. Gradients of $R^{2}>0.50$ are in bold.

\begin{tabular}{|c|c|c|c|c|c|c|c|c|c|c|}
\hline \multicolumn{11}{|c|}{ Downward shortwave solar radiation $K \downarrow\left(\mathrm{W} \mathrm{m}^{-2}\right)$} \\
\hline Site & $a_{\mathrm{N}}^{*}\left(\mathrm{~W}^{-1} \mathrm{~m}^{2}\right)$ & $R^{2}$ & $p$ & $a_{\mathrm{G}}$ & $R^{2}$ & $p$ & $a_{J}^{*}\left(\mathrm{~W}^{-1} \mathrm{~m}^{2}\right)$ & $R^{2}$ & $p$ & Average \\
\hline UKRU* & $1.21 \times 10^{-3}$ & 0.94 & $<0.001$ & $6.53 \times 10^{-5}$ & 0.11 & - & $6.28 \times 10^{-4}$ & 0.93 & $<0.001$ & 443 \\
\hline UKUB* & $6.81 \times 10^{-4}$ & 0.90 & $<0.001$ & $-8.26 \times 10^{-5}$ & 0.10 & - & $1.49 \times 10^{-4}$ & 0.19 & - & 448 \\
\hline UKRO* & $8.69 \times 10^{-4}$ & 0.98 & $<0.001$ & $-7.75 \times 10^{-6}$ & 0.00 & _ & $2.66 \times 10^{-4}$ & 0.64 & $<0.005$ & 464 \\
\hline DENRU & $2.22 \times 10^{-3}$ & 0.88 & $<0.001$ & $4.24 \times 10^{-4}$ & 0.20 & - & $1.38 \times 10^{-3}$ & 0.64 & $<0.001$ & 115 \\
\hline DENUB & $1.87 \times 10^{-3}$ & 0.91 & $<0.001$ & $1.47 \times 10^{-4}$ & 0.03 & - & $8.98 \times 10^{-4}$ & 0.48 & $<0.01$ & 115 \\
\hline DENRO & $2.46 \times 10^{-3}$ & 0.95 & $<0.001$ & $1.27 \times 10^{-4}$ & 0.01 & - & $6.77 \times 10^{-4}$ & 0.50 & $<0.005$ & 117 \\
\hline GERRU & $2.87 \times 10^{-3}$ & 0.98 & $<0.001$ & $9.88 \times 10^{-4}$ & 0.72 & $<0.01$ & $1.45 \times 10^{-3}$ & 0.81 & $<0.001$ & 130 \\
\hline GERUB & $3.18 \times 10^{-3}$ & 0.97 & $<0.001$ & $7.28 \times 10^{-4}$ & 0.51 & $<0.005$ & $1.53 \times 10^{-3}$ & 0.69 & $<0.001$ & 114 \\
\hline GERRO & $2.40 \times 10^{-3}$ & 0.95 & $<0.001$ & $-5.89 \times 10^{-4}$ & 0.09 & - & $9.95 \times 10^{-4}$ & 0.59 & $<0.005$ & 114 \\
\hline FINRU & $2.63 \times 10^{-3}$ & 0.76 & $<0.001$ & $1.01 \times 10^{-3}$ & 0.57 & $<0.01$ & $2.04 \times 10^{-3}$ & 0.82 & $<0.001$ & 91.5 \\
\hline FINUB & $1.38 \times 10^{-3}$ & 0.37 & - & $1.81 \times 10^{-4}$ & 0.08 & - & $8.99 \times 10^{-4}$ & 0.25 & - & 111 \\
\hline FINRO & $1.76 \times 10^{-3}$ & 0.59 & $<0.005$ & $9.15 \times 10^{-4}$ & 0.34 & $<0.005$ & $4.45 \times 10^{-4}$ & 0.03 & - & 114 \\
\hline SPARU & $3.46 \times 10^{-4}$ & 0.35 & $<0.05$ & $5.68 \times 10^{-4}$ & 0.13 & - & $1.97 \times 10^{-3}$ & 0.74 & $<0.001$ & 162 \\
\hline SPAUB & $5.92 \times 10^{-4}$ & 0.58 & $<0.05$ & $6.98 \times 10^{-4}$ & 0.23 & - & $1.58 \times 10^{-3}$ & 0.81 & $<0.001$ & 180 \\
\hline GRERU & $4.10 \times 10^{-4}$ & 0.52 & $<0.001$ & $7.14 \times 10^{-4}$ & 0.55 & $<0.001$ & $-6.30 \times 10^{-4}$ & 0.05 & - & 201 \\
\hline GREUB & $3.49 \times 10^{-4}$ & 0.31 & - & $-1.10 \times 10^{-4}$ & 0.02 & - & $8.97 \times 10^{-4}$ & 0.34 & $<0.05$ & 183 \\
\hline \multicolumn{11}{|c|}{ Relative humidity (\%) } \\
\hline Site & $a_{\mathrm{N}}^{*}\left(\%^{-1}\right)$ & $R^{2}$ & $p$ & $a_{\mathrm{G}}$ & $R^{2}$ & $p$ & $a_{J}^{*}\left(\%^{-1}\right)$ & $R^{2}$ & $p$ & Average \\
\hline UKRU & $-5.89 \times 10^{-2}$ & 0.85 & $<0.001$ & $1.69 \times 10^{-3}$ & 0.02 & - & $-3.35 \times 10^{-2}$ & 0.85 & $<0.001$ & 79.7 \\
\hline UKUB & $-3.42 \times 10^{-2}$ & 0.94 & $<0.001$ & $8.23 \times 10^{-3}$ & 0.24 & - & $-5.66 \times 10^{-3}$ & 0.19 & - & 75.3 \\
\hline UKRO & $-5.09 \times 10^{-2}$ & 0.85 & $<0.001$ & $7.03 \times 10^{-3}$ & 0.25 & - & $-1.49 \times 10^{-2}$ & 0.46 & $<0.05$ & 74.5 \\
\hline DENRU & $-3.90 \times 10^{-2}$ & 0.95 & $<0.001$ & $9.42 \times 10^{-3}$ & 0.74 & $<0.001$ & $5.45 \times 10^{-4}$ & 0.00 & - & 75.7 \\
\hline DENUB & $-3.14 \times 10^{-2}$ & 0.94 & $<0.001$ & $3.64 \times 10^{-3}$ & 0.06 & - & $2.57 \times 10^{-3}$ & 0.00 & - & 75.7 \\
\hline DENRO & $-3.64 \times 10^{-2}$ & 0.95 & $<0.001$ & $-1.21 \times 10^{-2}$ & 0.22 & - & $-3.91 \times 10^{-3}$ & 0.10 & - & 75.7 \\
\hline GERRU & $-5.08 \times 10^{-2}$ & 0.88 & $<0.001$ & $-1.30 \times 10^{-2}$ & 0.72 & $<0.001$ & $-2.46 \times 10^{-2}$ & 0.91 & $<0.001$ & 81.9 \\
\hline GERUB & $-5.35 \times 10^{-2}$ & 0.86 & $<0.001$ & $-6.34 \times 10^{-3}$ & 0.67 & $<0.001$ & $-2.25 \times 10^{-2}$ & 0.86 & $<0.001$ & 78.7 \\
\hline GERRO & $-2.83 \times 10^{-2}$ & 0.90 & $<0.001$ & $3.98 \times 10^{-3}$ & 0.05 & - & $-1.72 \times 10^{-2}$ & 0.81 & $<0.001$ & 78.7 \\
\hline FINRU & $-4.48 \times 10^{-2}$ & 0.94 & $<0.001$ & $-7.07 \times 10^{-3}$ & 0.65 & $<0.001$ & $-2.16 \times 10^{-2}$ & 0.87 & $<0.001$ & 80.1 \\
\hline FINUB & $-5.89 \times 10^{-2}$ & 0.95 & $<0.001$ & $1.04 \times 10^{-2}$ & 0.26 & - & $-6.52 \times 10^{-3}$ & 0.18 & - & 76.5 \\
\hline FINRO & $-3.34 \times 10^{-2}$ & 0.92 & $<0.001$ & $-1.47 \times 10^{-3}$ & 0.01 & - & $7.39 \times 10^{-3}$ & 0.10 & - & 71.1 \\
\hline SPARU & $-1.54 \times 10^{-2}$ & 0.90 & $<0.001$ & $-4.67 \times 10^{-3}$ & 0.08 & - & $-7.12 \times 10^{-3}$ & 0.14 & - & 66.4 \\
\hline SPAUB & $-4.84 \times 10^{-2}$ & 0.93 & $<0.001$ & $2.43 \times 10^{2}$ & 0.50 & $<0.01$ & $-9.83 \times 10^{-3}$ & 0.19 & - & 69.2 \\
\hline GRERU & $-7.72 \times 10^{-3}$ & 0.22 & - & $1.06 \times 10^{-2}$ & 0.06 & - & $-1.83 \times 10^{-1}$ & 0.15 & - & 70.0 \\
\hline GREUB & $-1.42 \times 10^{-2}$ & 0.62 & $<0.001$ & $2.83 \times 10^{-3}$ & 0.06 & - & $4.85 \times 10^{-4}$ & 0.00 & - & 60.5 \\
\hline
\end{tabular}

\subsubsection{Relative humidity}

Relative humidity is considered to have a negative effect on the occurrence of NPF events (Jeong et al., 2010; Hamed et al., 2011; Park et al., 2015; Dada et al., 2017; Li et al., 2019). While water in the atmosphere is one of the main compounds needed for the formation of the initial clusters either on the binary or ternary nucleation theory (Henschel et al., 2016; Korhonen et al., 1999; Mirabel and Katz, 1974), under atmospheric conditions it may also play a negative role in suppressing the number concentrations of new particles by increasing aerosol surface area (Li et al., 2019). Consistent with this, a negative relationship of the RH with NPF frequency was found for all the sites in this study, with very high $R^{2}$ for almost all of them $\left(R^{2}>0.80\right)$. This is not simple to interpret as solar radiation intensity, temperature, $\mathrm{RH}$, and CS are not independent variables, since an increase in the temperature of an air mass due to increased solar radiation will be associated with reduced $\mathrm{RH}$, which in turn affects the CS. The sites in Greece presented lower $R^{2}$ compared to the other sites, while GRERU was found to have the weakest correlation $\left(R^{2}=0.22\right)$. This may be due to the different sea- 
Table 3. Continued.

\begin{tabular}{|c|c|c|c|c|c|c|c|c|c|c|}
\hline \multicolumn{11}{|c|}{ Temperature $\left({ }^{\circ} \mathrm{C}\right)$} \\
\hline Site & $a_{\mathrm{N}}^{*}\left({ }^{\circ} \mathrm{C}^{-1}\right)$ & $R^{2}$ & $p$ & $a_{\mathrm{G}}$ & $R^{2}$ & $p$ & $a_{J}^{*}\left({ }^{\circ} \mathrm{C}^{-1}\right)$ & $R^{2}$ & $p$ & Average \\
\hline UKRU & $1.10 \times 10^{-1}$ & 0.93 & $<0.001$ & $7.85 \times 10^{-2}$ & 0.94 & $<0.001$ & $8.72 \times 10^{-2}$ & 0.84 & $<0.001$ & 10.6 \\
\hline UKUB & $9.04 \times 10^{-2}$ & 0.98 & $<0.001$ & $1.39 \times 10^{-1}$ & 0.96 & $<0.001$ & $6.34 \times 10^{-2}$ & 0.73 & $<0.005$ & 11.8 \\
\hline UKRO & $8.22 \times 10^{-2}$ & 0.98 & $<0.001$ & $3.51 \times 10^{-2}$ & 0.52 & $<0.05$ & $4.32 \times 10^{-2}$ & 0.44 & $<0.05$ & 12.1 \\
\hline DENRU & $6.68 \times 10^{-2}$ & 0.83 & $<0.001$ & $1.54 \times 10^{-2}$ & 0.08 & - & $6.68 \times 10^{-2}$ & 0.92 & $<0.001$ & 9.80 \\
\hline DENUB & $2.50 \times 10^{-2}$ & 0.45 & $<0.05$ & $2.40 \times 10^{-2}$ & 0.33 & - & $3.05 \times 10^{-2}$ & 0.45 & $<0.05$ & 9.82 \\
\hline DENRO & $6.64 \times 10^{-2}$ & 0.88 & $<0.001$ & $3.51 \times 10^{-3}$ & 0.00 & - & $2.96 \times 10^{-2}$ & 0.58 & $<0.005$ & 10.0 \\
\hline GERRU & $7.27 \times 10^{-2}$ & 0.92 & $<0.001$ & $5.65 \times 10^{-2}$ & 0.92 & $<0.001$ & $5.37 \times 10^{-2}$ & 0.93 & $<0.001$ & 10.3 \\
\hline GERUB & $8.20 \times 10^{-2}$ & 0.93 & $<0.001$ & $3.38 \times 10^{-2}$ & 0.62 & $<0.001$ & $4.28 \times 10^{-2}$ & 0.54 & $<0.005$ & 11.1 \\
\hline GERRO & $5.08 \times 10^{-2}$ & 0.89 & $<0.001$ & $-3.33 \times 10^{-3}$ & 0.00 & - & $1.61 \times 10^{-2}$ & 0.11 & - & 11.1 \\
\hline FINRU & $-2.01 \times 10^{-2}$ & 0.17 & - & $1.13 \times 10^{-1}$ & 0.79 & $<0.001$ & $4.27 \times 10^{-2}$ & 0.72 & $<0.001$ & 4.79 \\
\hline FINUB & $-4.21 \times 10^{-3}$ & 0.00 & - & $7.42 \times 10^{-2}$ & 0.83 & $<0.001$ & $1.67 \times 10^{-2}$ & 0.28 & - & 6.52 \\
\hline FINRO & $6.24 \times 10^{-2}$ & 0.65 & $<0.005$ & $9.28 \times 10^{-2}$ & 0.87 & $<0.001$ & $-1.09 \times 10^{-2}$ & 0.05 & - & 7.72 \\
\hline SPARU & $-2.51 \times 10^{-2}$ & 0.41 & $<0.05$ & $1.23 \times 10^{-1}$ & 0.92 & $<0.001$ & $9.11 \times 10^{-2}$ & 0.71 & $<0.001$ & 13.9 \\
\hline SPAUB & $-3.43 \times 10^{-3}$ & 0.02 & - & $6.67 \times 10^{-2}$ & 0.66 & $<0.005$ & $1.18 \times 10^{-2}$ & 0.08 & - & 18.2 \\
\hline GRERU & $-4.66 \times 10^{-2}$ & 0.75 & $<0.001$ & $1.74 \times 10^{-1}$ & 0.75 & $<0.001$ & $-9.45 \times 10^{-2}$ & 0.47 & $<0.05$ & 18.2 \\
\hline GREUB & $-1.00 \times 10^{-2}$ & 0.25 & - & $4.67 \times 10^{-2}$ & 0.62 & $<0.005$ & $-2.85 \times 10^{-2}$ & 0.20 & - & 17.6 \\
\hline \multicolumn{11}{|c|}{ Wind speed $\left(\mathrm{m} \mathrm{s}^{-1}\right)$} \\
\hline Site & $a_{\mathrm{N}}^{*}\left(\mathrm{~m}^{-1} \mathrm{~s}\right)$ & $R^{2}$ & $p$ & $a_{\mathrm{G}}$ & $R^{2}$ & $p$ & $a_{J}^{*}\left(\mathrm{~m}^{-1} \mathrm{~s}\right)$ & $R^{2}$ & $p$ & Average \\
\hline $\mathrm{UI}$ & $5.72 \times 10^{-2}$ & 0.20 & - & $-3.04 \times 10^{-2}$ & 0.07 & - & $\times 10^{-3}$ & 0.00 & - & 3.96 \\
\hline UKUB & $1.72 \times 10^{-1}$ & 0.87 & $<0.001$ & $-1.91 \times 10^{-1}$ & 0.71 & $<0.001$ & $3.56 \times 10^{-3}$ & 0.00 & - & 4.16 \\
\hline UKRO & $6.34 \times 10^{-2}$ & 0.19 & - & $3.21 \times 10^{-2}$ & 0.02 & - & $7.28 \times 10^{-2}$ & 0.45 & $<0.005$ & 4.14 \\
\hline DENRU & $1.08 \times 10^{-1}$ & 0.88 & $<0.001$ & $-2.33 \times 10^{-1}$ & 0.74 & $<0.001$ & $1.28 \times 10^{-1}$ & 0.44 & $<0.01$ & 4.17 \\
\hline DENUB & $1.50 \times 10^{-1}$ & 0.90 & $<0.001$ & $-3.33 \times 10^{-2}$ & 0.10 & - & $8.31 \times 10^{-2}$ & 0.19 & - & 4.17 \\
\hline DENRO & $1.65 \times 10^{-1}$ & 0.89 & $<0.001$ & $-1.51 \times 10^{-1}$ & 0.49 & $<0.001$ & $9.08 \times 10^{-3}$ & 0.00 & - & 4.16 \\
\hline GERRU & $-1.06 \times 10^{-1}$ & 0.57 & $<0.005$ & $-2.26 \times 10^{-1}$ & 0.83 & $<0.001$ & $-5.32 \times 10^{-3}$ & 0.00 & - & 2.58 \\
\hline GERUB & $-1.27 \times 10^{-1}$ & 0.52 & $<0.01$ & $-1.41 \times 10^{-1}$ & 0.60 & $<0.005$ & $-3.32 \times 10^{-2}$ & 0.04 & - & 2.33 \\
\hline GERRO & $-2.40 \times 10^{-1}$ & 0.56 & - & $-2.54 \times 10^{-1}$ & 0.38 & - & $-1.30 \times 10^{-1}$ & 0.22 & - & 2.33 \\
\hline FINRU & $1.62 \times 10^{-1}$ & 0.63 & $<0.005$ & $-1.29 \times 10^{-1}$ & 0.16 & $<0.05$ & $7.99 \times 10^{-2}$ & 0.07 & - & 1.31 \\
\hline FINUB & $-3.17 \times 10^{-2}$ & 0.08 & - & $7.26 \times 10^{-2}$ & 0.20 & $<0.05$ & $-9.74 \times 10^{-2}$ & 0.17 & - & 3.43 \\
\hline FINRO & $8.62 \times 10^{-2}$ & 0.51 & $<0.05$ & $-1.60 \times 10^{-1}$ & 0.32 & $<0.05$ & $-1.86 \times 10^{-1}$ & 0.32 & - & 4.26 \\
\hline SPARU & $-2.20 \times 10^{-2}$ & 0.02 & - & $3.80 \times 10^{-1}$ & 0.31 & - & $5.74 \times 10^{-2}$ & 0.02 & - & 0.94 \\
\hline SPAUB & $2.90 \times 10^{-1}$ & 0.93 & $<0.001$ & $7.71 \times 10^{-2}$ & 0.24 & - & $-5.90 \times 10^{-2}$ & 0.05 & - & 2.05 \\
\hline GRERU & $4.37 \times 10^{-2}$ & 0.54 & $<0.001$ & $1.01 \times 10^{-1}$ & 0.36 & $<0.005$ & $1.73 \times 10^{-3}$ & 0.00 & - & 6.06 \\
\hline GREUB & $-1.13 \times 10^{-1}$ & 0.47 & $<0.01$ & $-1.88 \times 10^{-1}$ & 0.50 & $<0.005$ & $-3.78 \times 10^{-2}$ & 0.01 & - & 1.87 \\
\hline
\end{tabular}

sonality of the events found for the Greek sites (being more balanced within a year), as there was an increased frequency of NPF events for the seasons with higher RH compared to other sites, making it a less important factor for their occurrence, as found in a previous study by Bousiotis et al. (2020). The growth rate, on the other hand, had a variable relationship, either positive or negative, with only a handful of background sites having strong correlations. The German background sites and FINRU, which were among the sites with the highest average RH (average RH for GERRU is $81.9 \%$, GERUB is $78.7 \%$, and FINUB is $80.1 \%$ ), presented a negative relationship between the RH and growth rate. DENRU (average $\mathrm{RH}$ at $75.7 \%$ ) had a positive relationship, which might indicate that the relationship between these two variables varies depending upon the RH range. The formation rate also appears to have a negative relationship with the $\mathrm{RH}$, though this relationship was significant $\left(R^{2}>0.40\right)$ for only six sites, which once again in most cases are sites with higher $\mathrm{RH}$ average conditions. Along with the results of the growth rate this might indicate that the RH becomes a more important factor in the development of NPF events as its values increase.

The normalised gradients once again provide some additional information. Regarding the NPF frequency, it is found that the $a_{\mathrm{N}}^{*}$ was more negative at rural sites compared to roadside sites. This indicates that the RH has a smaller ef- 
Table 3. Continued.

\begin{tabular}{|c|c|c|c|c|c|c|c|c|c|c|}
\hline \multicolumn{11}{|c|}{ Atmospheric pressure (mbar) } \\
\hline Site & $a_{\mathrm{N}}^{*}\left(\mathrm{mbar}^{-1}\right)$ & $R^{2}$ & $p$ & $a_{\mathrm{G}}$ & $R^{2}$ & $p$ & $a_{J}^{*}\left(\mathrm{mbar}^{-1}\right)$ & $R^{2}$ & $p$ & Average \\
\hline UKRU & $4.26 \times 10^{-2}$ & 0.83 & $<0.005$ & $3.93 \times 10^{-2}$ & 0.58 & $<0.005$ & $2.95 \times 10^{-2}$ & 0.47 & $<0.05$ & 1007.7 \\
\hline UKUB & $1.90 \times 10^{-2}$ & 0.50 & - & $1.17 \times 10^{-2}$ & 0.05 & $<0.05$ & $4.16 \times 10^{-3}$ & 0.04 & - & 1011.7 \\
\hline UKRO & $6.33 \times 10^{-2}$ & 0.95 & $<0.001$ & $-1.21 \times 10^{-1}$ & 0.40 & - & $-2.98 \times 10^{-2}$ & 0.17 & - & 1012 \\
\hline GERRU & $5.10 \times 10^{-2}$ & 0.97 & - & $8.95 \times 10^{-2}$ & 0.85 & $<0.001$ & $2.16 \times 10^{-2}$ & 0.21 & - & 1007.0 \\
\hline GERUB & $6.27 \times 10^{-2}$ & 0.97 & - & $4.00 \times 10^{-2}$ & 0.76 & - & $2.00 \times 10^{-2}$ & 0.37 & $<0.05$ & 995.5 \\
\hline GERRO & $4.57 \times 10^{-2}$ & 0.79 & - & $-9.61 \times 10^{-2}$ & 0.43 & - & $-2.80 \times 10^{-2}$ & 0.21 & - & 995.5 \\
\hline FINRU & $3.46 \times 10^{-2}$ & 0.88 & $<0.001$ & $2.90 \times 10^{-2}$ & 0.57 & $<0.001$ & $1.05 \times 10^{-2}$ & 0.14 & - & 985.1 \\
\hline FINUB & $2.61 \times 10^{-2}$ & 0.55 & $<0.005$ & $-3.57 \times 10^{-3}$ & 0.02 & - & $4.38 \times 10^{-3}$ & 0.05 & - & 1004.4 \\
\hline FINRO & $4.91 \times 10^{-2}$ & 0.70 & - & $-2.67 \times 10^{-2}$ & 0.17 & - & $1.43 \times 10^{-2}$ & 0.26 & - & 1008.8 \\
\hline SPARU & $-2.02 \times 10^{-2}$ & 0.09 & - & $4.79 \times 10^{-2}$ & 0.14 & - & $2.89 \times 10^{-2}$ & 0.08 & - & 939.3 \\
\hline SPAUB & $-2.83 \times 10^{-2}$ & 0.44 & $<0.05$ & $1.86 \times 10^{-2}$ & 0.08 & - & $1.68 \times 10^{-2}$ & 0.21 & - & 1006.3 \\
\hline GRERU & $6.00 \times 10^{-2}$ & 0.46 & $<0.001$ & $-1.50 \times 10^{-1}$ & 0.73 & - & $8.14 \times 10^{-2}$ & 0.33 & - & 1014.5 \\
\hline GREUB & $9.42 \times 10^{-3}$ & 0.10 & $<0.05$ & $-1.00 \times 10^{-1}$ & 0.71 & - & $1.58 \times 10^{-2}$ & 0.04 & - & 1015.7 \\
\hline
\end{tabular}

* Global solar irradiation measurements in $\mathrm{kJ} \mathrm{m}^{-2}$.

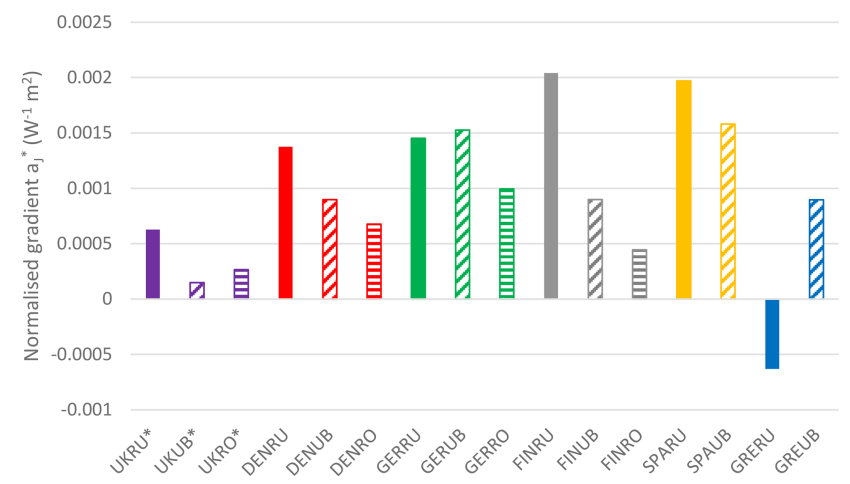

Figure 3. Normalised gradients $a_{J}^{*}$ for $K \downarrow$ (*UK sites are calculated with solar irradiance).

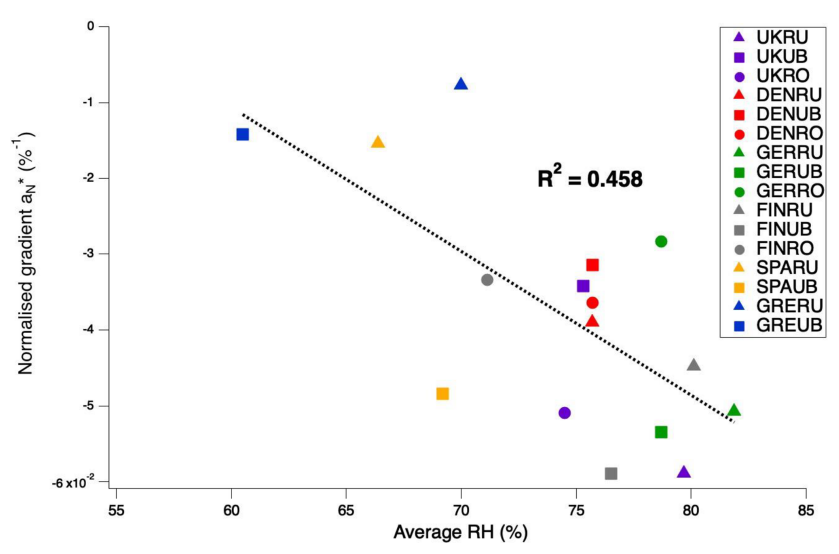

Figure 4. Relationship of average relative humidity and normalised gradients $a_{\mathrm{N}}^{*}$. fect at roadside sites, as other variables, such as the atmospheric composition, are probably more important within the complex environment at this type of site. Additionally, the relationship between $a_{\mathrm{N}}^{*}$ and average RH at the sites had a negative relationship $\left(R^{2}=0.46\right)$, which further shows that the RH becomes a more important factor at higher values (Fig. 4). Furthermore, at the rural and roadside sites with $R^{2}$ higher than 0.40 for the relation between $\mathrm{RH}$ and the formation rate (UK and German sites), it was found that the $a_{J}^{*}$ was more negative at the rural sites, which indicates that the $\mathrm{RH}$ is a more important factor at rural sites compared to their respective roadside sites.

\subsubsection{Temperature}

Temperature can have both a direct and indirect effect on the development of NPF events, as it is directly associated with the abundance of both biogenic and anthropogenic volatile carbon, which is an important group of compounds whose oxidation products can participate in nucleation itself (Lehtipalo et al., 2018; Rose et al., 2018) and in the growth of newly formed particles. It may also have a negative effect on particle size distributions or number concentrations through other processes such as particle evaporation. Most of the sites in the present study presented a strong relationship of NPF frequency with temperature, which in most cases was positive, though in many cases (such as the Danish, Finnish, and Spanish sites - Fig. S2b, d, and e) there seems to be a peak in the NPF frequency at some temperature, after which a decline starts (though being at the higher end, it does not greatly affect the results). Sites with smaller $R^{2}$ (weaker association with temperature) were mainly those that have a seasonal variation favouring seasons other than summer. These sites not only had a weaker relationship of NPF frequency 
with temperature, but in most cases there was a negative relationship (background sites in Finland, Spain, and Greece). The Finnish sites, having the lowest average temperatures and a sufficient amount of data for temperatures below zero, show at all three sites the possible presence of a peak in the NPF event frequency for temperatures below zero (Fig. S2d). This seems to be the cause of the weak relationships found there, and they seem to be associated with the formation rate $J_{10}$, which also seems to have an increasing trend below $0^{\circ} \mathrm{C}$ (Fig. S2p). This may depend on the nucleation mechanism occurring, as cluster evaporation rates of sulfuric acid clusters are sensitive to the ternary stabilising compound present (Olenius et al., 2017) and the possible enhancement of growth mechanisms at lower temperatures (below $5^{\circ} \mathrm{C}$ ) by other chemical compounds in the atmosphere (i.e. nitric acid and ammonia), as found by Wang et al. (2020). Laboratory experiments show that the characteristics of organic aerosol forming from alpha-pinene is governed by gas-phase oxidation (e.g. Ye et al., 2018). In the real atmosphere, the higher temperature enhances the amount of biogenic vapour (e.g. Paasonen et al., 2013), and although oxidation can be more efficient at higher temperatures, lower temperatures favour the formation of more non-volatile compounds (Quéléver et al., 2019; Stolzenburg et al., 2018; Ye et al., 2018).

Growth rate had a more uniform trend, with almost all sites having a positive relationship with temperature (apart from GERRO, though with $R^{2}=0.00$ ). This relationship was very strong for most sites $\left(R^{2}>0.60\right.$ for 10 sites), which also confirms the summer peak found for the growth rate at most of these sites in other studies (Bousiotis et al., 2020, 2019). A rather strong relationship $\left(R^{2}>0.50\right)$ with temperature was also found for the formation rate for most sites, which was positive for almost all sites (apart from FINRO with $R^{2}=0.01$ and the Greek sites with $\left.R^{2}<0.47\right)$. As with the NPF frequency, in general the sites with a seasonal variation of events that favoured summer had the strongest relationship (high $R^{2}$ ) of temperature with formation rate, which might indicate that this variable, either through its direct or indirect effect, is an important one for the seasonal variability of NPF events in a given area.

The normalised gradients for this variable did not present a clear trend among the areas studied, other than presenting greater $a_{\mathrm{N}}^{*}$ for the sites with a summer peak in their NPF event seasonal variation. As with other meteorological variables, the importance of this variable became smaller with increased values in the average conditions for both the NPF frequency (Fig. 5) and $J_{10}$, though these relationships were not significant (biased by the very low average temperatures and different behaviour of the variables at the Finnish sites, without which the relationship becomes a lot clearer, as indicated in Fig. S13). The variation within the sites of the same area (different sites in same country or region) appears to directly follow the variability of temperature, showing that the temperature directly affects the occurrence of NPF events when other meteorological factors remain constant, having a

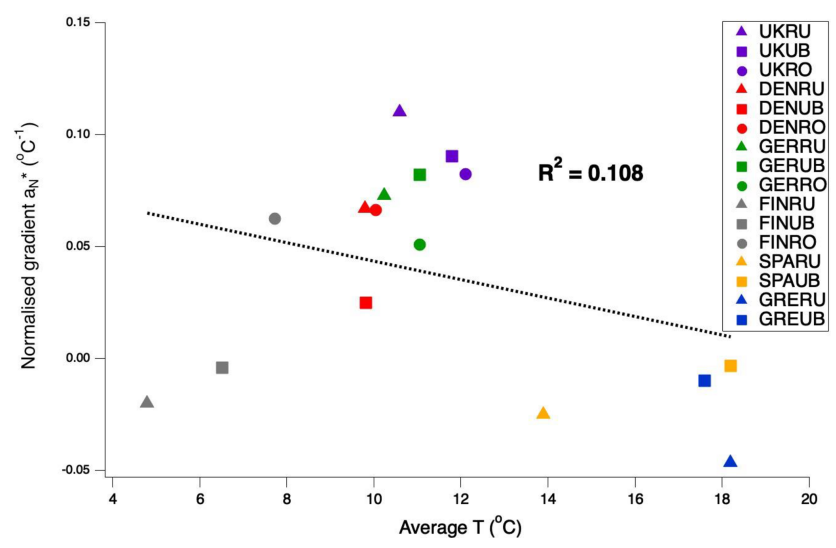

Figure 5. Relationship of average temperature and normalised gradients $a_{\mathrm{N}}^{*}$.

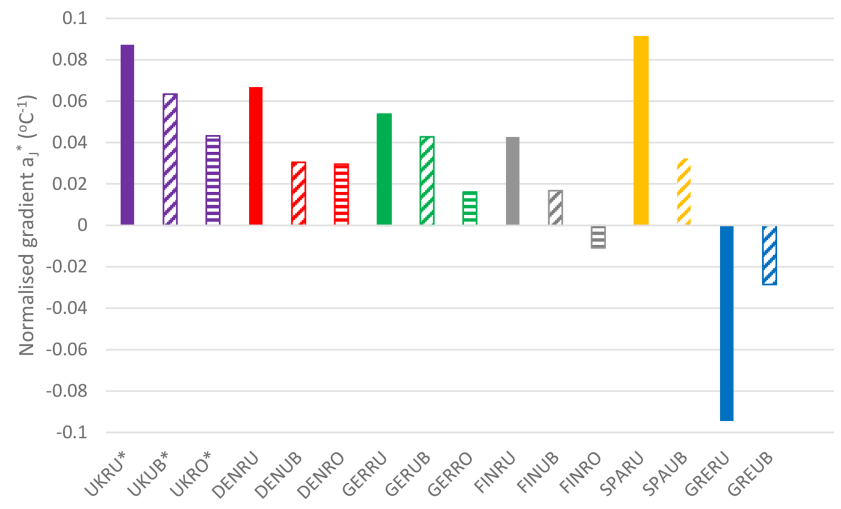

Figure 6. Normalised gradients $a_{J}^{*}$ for temperature.

negative trend for all countries but Finland. The $a_{J}^{*}$, however, is found to be greater (positively or negatively) at the rural background sites than at the other two types of sites at all areas studied, showing that it is a more important factor for the formation rate at this type of site compared to others (Fig. 6).

\subsubsection{Wind speed}

Wind speed may have both a positive and a negative effect on the occurrence of NPF events. On one hand, it may promote NPF events through the increased mixing of condensable compounds in the atmosphere and by reducing the CS. On the other hand, high wind speeds may suppress NPF events due to increased dilution. It should be considered that the variability found is also affected by the specific conditions found at each site. The wind speed measurements in many cases, especially at urban sites, can be biased by the local topography or specific conditions found at each site, thus representing the local conditions for this variable rather than the regional ones. Similarly, measurements of wind speed at well-sited meteorological stations may be 
more representative of regional conditions than of those affecting the sites of nucleation measurement. The sites in this study presented mixed results for both the importance and the effect of the wind speed variability. Three different behaviours were found in the variation of NPF event frequency and wind speed, which appear to be associated with local conditions as they are almost uniformly found among the sites within close proximity. Some sites presented a steady increase in NPF event frequency with wind speed (Danish sites - UKUB, FINRU, SPAUB, and GRERU), while others were found to steadily decline with increasing wind speeds (German sites - it should be noted that the German sites are the only ones that are located at a great distance from the sea), and some were found to reach a peak and then decline, which also leads to smaller $R^{2}$ (UKRU, UKRO, SPARU, and to a lesser extent GREUB - Fig. S4a, e, and f). The reasons for these differences between the sites are very hard to distinguish as apart from the wind speed the origin and the characteristics of these air masses play a crucial role. Following this, it appears that NPF frequency is very low or zero for wind speeds close to calm for the sites with an increasing trend (as well as those that have a peak and decline after), while the opposite is observed for the German sites where the maximum NPF frequency is found for very low wind speeds (Fig. S4c).

Similarly, the effect of different wind speeds upon the growth rate also varied a lot, though it was found to be negative in all the cases in which $R^{2}$ was higher than 0.50 (UKUB, DENRU, DENRO, GERRU, GERUB, and GREUB). Finally, the formation rate was found to have a significant correlation $\left(R^{2}>0.40\right)$ only at two sites (UKRO and DENRU), probably indicating that the variability of the wind speed either does not affect this variable or its effect is rather small.

The normalised gradients did not have any notable relationship with either the NPF frequency or the formation rate, further confirming that the effect of the different wind speeds is not due to its variability only, but it is also influenced by the characteristics of the incoming air masses and specific local conditions found at each site.

\subsubsection{Pressure}

At almost all the sites with available data (apart from the Spanish), the NPF frequency presented a positive relationship with high significance at all types of sites. The greater significance found at the rural sites (apart from SPARU) indicates the increased importance of meteorological conditions for the occurrence of NPF events at this type of site. The growth rate also presented a similar picture, with positive relationships at all the background sites in this study except the ones in Greece $\left(R^{2}>0.71\right)$ and FINUB (though with low $R^{2}$ at 0.02 ). This is probably associated with the seasonal variation found in Greece where higher growth rates were found in summer, a period when increased wind speeds and lower atmospheric pressure were found due to the Etesian winds, which are part of a pressure system that develops in the region every summer (Kalkavouras et al., 2017). An interesting finding is the negative gradients at all the roadside sites, though the significance of these results is relatively low $\left(R^{2}<0.43\right)$ and always lower compared to the rural sites. The effects of pressure above are not likely to be important. Once again, however, this is not an independent variable, and higher pressure in summer tends to be associated with higher insolation and temperatures as well as lower RH. Since most events occur in the warmer months of the year, this is probably the explanation for the apparent effects of pressure. The formation rate presented relationships of low significance $\left(R^{2}<0.47\right)$ for the sites in this study. Due to this, pressure should not be an important factor for the formation rate at any type of site.

The normalised gradients did not present any clear trends, even for the NPF frequency for which the results presented significant relationships at almost all sites.

\subsection{Atmospheric composition}

The gradients, $R^{2}$, and $p$ values from the analysis of a number of air pollutants $\left(\mathrm{SO}_{2}, \mathrm{NO}_{x}, \mathrm{O}_{3}\right.$, organic compounds, sulfate, and ammonia) and the CS, as well as the average conditions of these variables, are found in Table 4. The results for each site and variable are found in Figs. S6-S12.

\subsubsection{Sulfur dioxide $\left.\mathrm{SO}_{2}\right)$}

Sulfur dioxide, as a precursor of $\mathrm{H}_{2} \mathrm{SO}_{4}$, is considered one of the main components associated with the NPF process. According to nucleation theories and observations, $\mathrm{H}_{2} \mathrm{SO}_{4}$ is the most important compound from which the initial clusters are formed, and it is also one of the candidate compounds for the initial steps of particle growth (Kirkby et al., 2011; Nieminen et al., 2010; Sipila et al., 2010; Stolzenburg et al., 2020). As $\mathrm{H}_{2} \mathrm{SO}_{4}$ in the atmosphere is produced from oxidation reactions of $\mathrm{SO}_{2}$ it would be expected that increased concentrations of the latter would be associated with increased values for all the variables associated with the NPF process. Contrary to this, though, the relationship of $\mathrm{SO}_{2}$ concentrations with NPF frequency was found to be negative at all the sites in this study with available data. This is expected as the average concentrations of $\mathrm{SO}_{2}$ on NPF event days were found to be lower compared to the average conditions in most cases, as found by Bousiotis et al. $(2019,2020)$. This relationship was relatively strong $\left(R^{2}>0.50\right)$ in most areas, with an increased significance at roadside sites compared to their respective rural sites. As this is a negative relationship, this may indicate that $\mathrm{SO}_{2}$ is in sufficient concentrations for $\mathrm{H}_{2} \mathrm{SO}_{4}$ formation, thus not suppressing the occurrence of NPF events, as well as showing that in increased concentrations, it is a more important factor (or surrogate for a factor) in preventing the occurrence of NPF events within 
Table 4. Normalised gradients (non-normalised for growth rate), $R^{2}$, and $p$ values ( - for values $>0.05$ ) for the relation between atmospheric composition variables and NPF event variables. Gradients of $R^{2}>0.50$ are in bold.

\begin{tabular}{|c|c|c|c|c|c|c|c|c|c|c|}
\hline \multicolumn{11}{|c|}{$\mathrm{SO}_{2}\left(\mu \mathrm{g} \mathrm{m}^{-3}\right)$} \\
\hline Site & $a_{\mathrm{N}}^{*}\left(\mu \mathrm{g}^{-1} \mathrm{~m}^{3}\right)$ & $R^{2}$ & $p$ & $a_{\mathrm{G}}$ & $R^{2}$ & $p$ & $a_{J}^{*}\left(\mu \mathrm{g}^{-1} \mathrm{~m}^{3}\right)$ & $R^{2}$ & $p$ & Average \\
\hline UKRU & $-1.97 \times 10^{-1}$ & 0.38 & $<0.05$ & $-6.17 \times 10^{-2}$ & 0.02 & - & $3.30 \times 10^{-1}$ & 0.06 & - & 1.64 \\
\hline UKUB & $-2.57 \times 10^{-1}$ & 0.62 & $<0.001$ & $1.93 \times 10^{-2}$ & 0.00 & - & $4.18 \times 10^{-1}$ & 0.40 & - & 2.04 \\
\hline UKRO & $-1.03 \times 10^{-1}$ & 0.82 & $<0.001$ & $6.90 \times 10^{-2}$ & 0.34 & $<0.01$ & $8.43 \times 10^{-2}$ & 0.77 & $<0.001$ & 7.46 \\
\hline DENRU & $-9.77 \times 10^{-1}$ & 0.53 & $<0.05$ & $2.84 \times 10^{0}$ & 0.37 & - & $4.38 \times 10^{-1}$ & 0.09 & - & 0.52 \\
\hline DENRO & $-4.20 \times 10^{-1}$ & 0.91 & $<0.001$ & $6.42 \times 10^{-1}$ & 0.54 & $<0.005$ & $5.66 \times 10^{-1}$ & 0.62 & $<0.001$ & 0.97 \\
\hline FINRU & $-5.66 \times 10^{-1}$ & 0.05 & - & $-1.42 \times 10^{0}$ & 0.19 & - & $-6.30 \times 10^{-2}$ & 0.00 & - & 0.09 \\
\hline SPARU & $-3.62 \times 10^{-1}$ & 0.74 & $<0.001$ & $-1.33 \times 10^{-1}$ & 0.02 & - & $-3.55 \times 10^{-2}$ & 0.01 & - & 0.95 \\
\hline SPAUB & $-2.93 \times 10^{-2}$ & 0.04 & - & $4.12 \times 10^{-1}$ & 0.59 & - & $1.07 \times 10^{-1}$ & 0.29 & - & 1.99 \\
\hline \multicolumn{11}{|c|}{$\mathrm{NO}_{x}$ or $\mathrm{NO}_{2}(\mathrm{ppb})$} \\
\hline Site & $a_{\mathrm{N}}^{*}\left(\mathrm{ppb}^{-1}\right)$ & $R^{2}$ & $p$ & $a_{\mathrm{G}}$ & $R^{2}$ & $p$ & $a_{J}^{*}\left(\mathrm{ppb}^{-1}\right)$ & $R^{2}$ & $p$ & Average \\
\hline UKRU & $-4.99 \times 10^{-2}$ & 0.67 & $<0.005$ & $4.52 \times 10^{-2}$ & 0.58 & $<0.05$ & $-4.51 \times 10^{-2}$ & 0.70 & $<0.005$ & 11.7 \\
\hline UKUB & $-8.75 \times 10^{-3}$ & 0.83 & $<0.001$ & $-3.97 \times 10^{-4}$ & 0.00 & - & $-1.09 \times 10^{-2}$ & 0.43 & $<0.05$ & 53.6 \\
\hline UKRO & $-3.22 \times 10^{-3}$ & 0.72 & $<0.001$ & $1.44 \times 10^{-3}$ & 0.39 & $<0.05$ & $2.19 \times 10^{-3}$ & 0.66 & $<0.001$ & 299 \\
\hline DENRU & $-9.41 \times 10^{-2}$ & 0.43 & $<0.005$ & $-4.89 \times 10^{-3}$ & 0.00 & $<0.001$ & $-6.47 \times 10^{-2}$ & 0.55 & $<0.01$ & 5.42 \\
\hline DENUB & $-4.99 \times 10^{-2}$ & 0.68 & $<0.001$ & $2.85 \times 10^{-2}$ & 0.26 & - & $8.55 \times 10^{-4}$ & 0.00 & - & 10.5 \\
\hline DENRO & $-5.10 \times 10^{-3}$ & 0.75 & $<0.001$ & $1.10 \times 10^{-2}$ & 0.69 & $<0.001$ & $8.33 \times 10^{-3}$ & 0.88 & $<0.001$ & 68.5 \\
\hline FINRU & $-7.27 \times 10^{-1}$ & 0.54 & $<0.001$ & $-2.74 \times 10^{-1}$ & 0.11 & - & $1.95 \times 10^{-1}$ & 0.05 & - & 0.72 \\
\hline FINRO & $-6.24 \times 10^{-3}$ & 0.68 & $<0.001$ & $1.70 \times 10^{-3}$ & 0.12 & - & $3.25 \times 10^{-3}$ & 0.03 & - & 88.1 \\
\hline SPARU ${ }^{\mathrm{a}}$ & $-1.53 \times 10^{-2}$ & 0.05 & - & $2.54 \times 10^{-2}$ & 0.01 & - & $1.25 \times 10^{-1}$ & 0.21 & - & 3.26 \\
\hline SPAUB $^{\mathrm{a}}$ & $-2.59 \times 10^{-2}$ & 0.62 & $<0.005$ & $2.23 \times 10^{-2}$ & 0.70 & $<0.001$ & $2.57 \times 10^{-3}$ & 0.01 & - & 31.4 \\
\hline GRERU $^{\mathrm{a}}$ & $3.01 \times 10^{-1}$ & 0.19 & - & $-1.40 \times 10^{0}$ & 0.75 & $<0.001$ & $5.23 \times 10^{-1}$ & 0.13 & - & 0.52 \\
\hline \multicolumn{11}{|l|}{$\mathrm{O}_{3}(\mathrm{ppb})$} \\
\hline Site & $a_{\mathrm{N}}^{*}\left(\mathrm{ppb}^{-1}\right)$ & $R^{2}$ & $p$ & $a_{\mathrm{G}}$ & $R^{2}$ & $p$ & $a_{J}^{*}\left(\mathrm{ppb}^{-1}\right)$ & $R^{2}$ & $p$ & Average \\
\hline UKRU & $2.27 \times 10^{-2}$ & 0.88 & $<0.001$ & $-4.89 \times 10^{-2}$ & 0.53 & $<0.005$ & $-3.53 \times 10^{-3}$ & 0.01 & - & 54.4 \\
\hline UKUB & $1.37 \times 10^{-2}$ & 0.87 & $<0.001$ & $-3.45 \times 10^{-2}$ & 0.68 & $<0.001$ & $-5.95 \times 10^{-3}$ & 0.05 & - & 39.3 \\
\hline UKRO & $7.46 \times 10^{-2}$ & 0.95 & $<0.001$ & $-1.06 \times 10^{-2}$ & 0.09 & - & $-2.44 \times 10^{-2}$ & 0.63 & $<0.005$ & 16.2 \\
\hline DENRU & $4.97 \times 10^{-2}$ & 0.92 & $<0.001$ & $-1.32 \times 10^{-2}$ & 0.15 & - & $1.23 \times 10^{-2}$ & 0.08 & - & 30.1 \\
\hline DENUB & $5.85 \times 10^{-2}$ & 0.84 & $<0.001$ & $-1.69 \times 10^{-2}$ & 0.58 & - & $2.77 \times 10^{-2}$ & 0.32 & $<0.05$ & 28.2 \\
\hline DENRO & $6.42 \times 10^{-2}$ & 0.51 & $<0.05$ & $1.39 \times 10^{-2}$ & 0.03 & - & $3.24 \times 10^{-2}$ & 0.91 & $<0.05$ & 31.1 \\
\hline FINRU & $6.76 \times 10^{-2}$ & 0.77 & $<0.05$ & $-4.23 \times 10^{-2}$ & 0.60 & - & $3.92 \times 10^{-2}$ & 0.37 & $<0.05$ & 27.4 \\
\hline FINRO & $2.38 \times 10^{-2}$ & 0.91 & $<0.001$ & $6.11 \times 10^{-3}$ & 0.24 & - & $-1.83 \times 10^{-2}$ & 0.29 & - & 37.1 \\
\hline SPARU & $1.57 \times 10^{-2}$ & 0.02 & - & $4.34 \times 10^{-2}$ & 0.11 & - & $1.31 \times 10^{-2}$ & 0.31 & - & 75.9 \\
\hline SPAUB & $7.99 \times 10^{-3}$ & 0.38 & $<0.05$ & $-5.83 \times 10^{-3}$ & 0.30 & - & $-1.13 \times 10^{-3}$ & 0.01 & - & 54.9 \\
\hline GRERU & $7.55 \times 10^{-3}$ & 0.04 & - & $3.68 \times 10^{-2}$ & 0.17 & - & $-3.01 \times 10^{-2}$ & 0.15 & - & 49.5 \\
\hline \multicolumn{11}{|c|}{ Particulate organic carbon $\left(\mu \mathrm{g} \mathrm{m}^{-3}\right)$} \\
\hline Site & $a_{\mathrm{N}}^{*}\left(\mu \mathrm{g}^{-1} \mathrm{~m}^{3}\right)$ & $R^{2}$ & $p$ & $a_{\mathrm{G}}$ & $R^{2}$ & $p$ & $a_{J}^{*}\left(\mu \mathrm{g}^{-1} \mathrm{~m}^{3}\right)$ & $R^{2}$ & $p$ & Average \\
\hline UKRU & $-3.30 \times 10^{-2}$ & 0.00 & - & $1.13 \times 10^{0}$ & 0.42 & $<0.005$ & $2.13 \times 10^{-1}$ & 0.16 & - & 1.96 \\
\hline UKUB & $-2.76 \times 10^{-1}$ & 0.59 & $<0.005$ & $6.63 \times 10^{-1}$ & 0.58 & $<0.05$ & $2.19 \times 10^{-1}$ & 0.55 & $<0.05$ & 3.63 \\
\hline UKRO & $-3.78 \times 10^{-1}$ & 0.89 & $<0.001$ & $8.12 \times 10^{-1}$ & 0.57 & $<0.005$ & $4.60 \times 10^{-1}$ & 0.75 & $<0.001$ & 6.24 \\
\hline DENRU & $-4.44 \times 10^{-1}$ & 0.75 & $<0.001$ & $2.24 \times 10^{-1}$ & 0.11 & - & $-3.17 \times 10^{-1}$ & 0.68 & $<0.01$ & 1.48 \\
\hline DENRO & $-7.80 \times 10^{-2}$ & 0.11 & - & $1.10 \times 10^{0}$ & 0.77 & $<0.005$ & $4.02 \times 10^{-1}$ & 0.81 & $<0.005$ & 2.59 \\
\hline GERRU & $-1.26 \times 10^{-1}$ & 0.24 & - & $1.35 \times 10^{-1}$ & 0.09 & - & $3.14 \times 10^{-2}$ & 0.03 & - & 2.18 \\
\hline FINRU & $2.27 \times 10^{-2}$ & 0.00 & - & $3.39 \times 10^{-1}$ & 0.60 & $<0.005$ & $-3.46 \times 10^{-1}$ & 0.16 & - & 1.78 \\
\hline GRERU & $-2.08 \times 10^{-1}$ & 0.11 & - & $7.87 \times 10^{-1}$ & 0.41 & $<0.05$ & $8.94 \times 10^{-1}$ & 0.11 & - & 1.58 \\
\hline
\end{tabular}


Table 4. Continued.

\begin{tabular}{|c|c|c|c|c|c|c|c|c|c|c|}
\hline \multicolumn{11}{|c|}{ Sulfate $\left(\mu \mathrm{g} \mathrm{m}^{-3}\right)$} \\
\hline Site & $a_{\mathrm{N}}^{*}\left(\mu \mathrm{g}^{-1} \mathrm{~m}^{3}\right)$ & $R^{2}$ & $p$ & $a_{\mathrm{G}}$ & $R^{2}$ & $p$ & $a_{J}^{*}\left(\mu \mathrm{g}^{-1} \mathrm{~m}^{3}\right)$ & $R^{2}$ & $p$ & Average \\
\hline UKRU $^{\mathrm{b}}$ & $-2.62 \times 10^{-1}$ & 0.57 & $<0.001$ & $7.34 \times 10^{-1}$ & 0.77 & $<0.001$ & $7.99 \times 10^{-1}$ & 0.44 & $<0.05$ & 1.97 \\
\hline $\mathrm{UKUB}^{\mathrm{b}}$ & $-3.57 \times 10^{-1}$ & 0.89 & $<0.001$ & $9.28 \times 10^{-1}$ & 0.44 & $<0.01$ & $9.72 \times 10^{-1}$ & 0.16 & - & 1.58 \\
\hline $\mathrm{UKRO}^{\mathrm{b}}$ & $-6.05 \times 10^{-2}$ & 0.24 & - & $3.04 \times 10^{-1}$ & 0.34 & $<0.05$ & $-6.22 \times 10^{-2}$ & 0.04 & - & 1.98 \\
\hline DENRU $^{\mathrm{c}}$ & $-7.81 \times 10^{-1}$ & 0.34 & $<0.05$ & $1.02 \times 10^{0}$ & 0.60 & $<0.05$ & $-1.03 \times 10^{0}$ & 0.63 & $<0.01$ & 0.52 \\
\hline DENRO $^{\mathrm{c}}$ & $-8.23 \times 10^{-1}$ & 0.28 & - & $1.99 \times 10^{0}$ & 0.22 & - & $2.82 \times 10^{-1}$ & 0.12 & - & 0.55 \\
\hline GERRU $^{\mathrm{b}}$ & $-3.37 \times 10^{-2}$ & 0.00 & - & $5.89 \times 10^{-1}$ & 0.11 & - & $-4.89 \times 10^{-2}$ & 0.01 & - & 0.92 \\
\hline FINRU $^{\mathrm{d}}$ & $-1.18 \times 10^{0}$ & 0.65 & $<0.001$ & $2.35 \times 10^{-1}$ & 0.09 & - & $-2.53 \times 10^{-1}$ & 0.17 & - & 1.02 \\
\hline \multicolumn{11}{|c|}{ Condensation sink $\left(\mathrm{s}^{-1}\right)$} \\
\hline Site & $a_{\mathrm{N}}^{*}(\mathrm{~s})$ & $R^{2}$ & $p$ & $a_{\mathrm{G}}$ & $R^{2}$ & $p$ & $a_{J}^{*}(\mathrm{~s})$ & $R^{2}$ & $p$ & Average \\
\hline UKRU & $-2.28 \times 10^{2}$ & 0.72 & $<0.001$ & $2.64 \times 10^{2}$ & 0.60 & $<0.001$ & $7.58 \times 10^{1}$ & 0.22 & - & $3.38 \times 10^{-3}$ \\
\hline UKUB & $-1.66 \times 10^{2}$ & 0.78 & $<0.001$ & $2.49 \times 10^{2}$ & 0.41 & $<0.05$ & $1.73 \times 10^{2}$ & 0.35 & $<0.05$ & $7.41 \times 10^{-3}$ \\
\hline UKRO & $-4.03 \times 10^{1}$ & 0.75 & $<0.001$ & $2.33 \times 10^{1}$ & 0.18 & - & $8.94 \times 10^{1}$ & 0.91 & $<0.001$ & $2.12 \times 10^{-2}$ \\
\hline DENRU & $-4.48 \times 10^{1}$ & 0.91 & $<0.001$ & $6.90 \times 10^{1}$ & 0.49 & $<0.05$ & $5.37 \times 10^{1}$ & 0.24 & - & $9.46 \times 10^{-3}$ \\
\hline DENUB & $-3.78 \times 10^{1}$ & 0.75 & $<0.001$ & $3.58 \times 10^{1}$ & 0.25 & - & $1.55 \times 10^{1}$ & 0.56 & $<0.005$ & $1.42 \times 10^{-2}$ \\
\hline DENRO & $-1.06 \times 10^{1}$ & 0.73 & $<0.001$ & $2.53 \times 10^{1}$ & 0.56 & $<0.005$ & $2.72 \times 10^{1}$ & 0.79 & $<0.001$ & $3.10 \times 10^{-2}$ \\
\hline GERRU & $1.54 \times 10^{2}$ & 0.86 & $<0.001$ & $1.33 \times 10^{2}$ & 0.56 & $<0.001$ & $6.67 \times 10^{1}$ & 0.63 & $<0.001$ & $7.02 \times 10^{-3}$ \\
\hline GERUB & $3.59 \times 10^{1}$ & 0.56 & $<0.005$ & $3.63 \times 10^{1}$ & 0.17 & - & $4.74 \times 10^{1}$ & 0.75 & $<0.001$ & $9.11 \times 10^{-3}$ \\
\hline GERRO & $3.89 \times 10^{1}$ & 0.22 & $<0.05$ & $-2.21 \times 10^{1}$ & 0.03 & $<0.005$ & $3.54 \times 10^{1}$ & 0.45 & $<0.005$ & $1.20 \times 10^{-2}$ \\
\hline FINRU & $-1.80 \times 10^{2}$ & 0.59 & $<0.005$ & $4.01 \times 10^{2}$ & 0.74 & $<0.001$ & $4.98 \times 10^{1}$ & 0.10 & - & $2.32 \times 10^{-3}$ \\
\hline FINUB & $-1.51 \times 10^{2}$ & 0.63 & $<0.005$ & $8.14 \times 10^{1}$ & 0.31 & - & $2.01 \times 10^{2}$ & 0.41 & $<0.05$ & $6.34 \times 10^{-3}$ \\
\hline FINRO & $-6.99 \times 10^{1}$ & 0.77 & $<0.001$ & $-1.56 \times 10^{1}$ & 0.05 & - & $2.42 \times 10^{2}$ & 0.83 & $<0.001$ & $8.96 \times 10^{-3}$ \\
\hline SPARU & $-2.15 \times 10^{2}$ & 0.65 & $<0.005$ & $1.86 \times 10^{1}$ & 0.00 & - & $8.60 \times 10^{1}$ & 0.47 & $<0.05$ & $5.49 \times 10^{-3}$ \\
\hline SPAUB & $-1.18 \times 10^{2}$ & 0.65 & $<0.005$ & $3.74 \times 10^{1}$ & 0.38 & $<0.05$ & $9.51 \times 10^{1}$ & 0.52 & $<0.01$ & $1.00 \times 10^{-2}$ \\
\hline GRERU & $4.33 \times 10^{0}$ & 0.00 & - & $2.86 \times 10^{2}$ & 0.70 & $<0.001$ & $1.77 \times 10^{2}$ & 0.56 & $<0.005$ & $4.66 \times 10^{-3}$ \\
\hline GREUB & $1.64 \times 10^{2}$ & 0.65 & $<0.001$ & $9.31 \times 10^{1}$ & 0.28 & $<0.05$ & $1.73 \times 10^{2}$ & 0.83 & $<0.001$ & $7.55 \times 10^{-3}$ \\
\hline
\end{tabular}

${ }^{\mathrm{a}} \mathrm{NO}_{2}$ measurements; ${ }^{\mathrm{b}}$ measurements in $\mathrm{PM}_{10} ;{ }^{\mathrm{c}}$ measurements in $\mathrm{PM}_{2.5} ;{ }^{\mathrm{d}}$ measurements in $\mathrm{PM}_{1}$.

the urban environment, as higher $\mathrm{SO}_{2}$ is likely associated with increased co-emitted particle pollution and hence CS. The growth rate, on the other hand, presented mixed results, and the significance of the relationships is low in most cases, which makes these results unreliable. Finally, the relationship of $\mathrm{SO}_{2}$ concentrations with the formation rate was found to be positive at all sites but SPARU and FINRU (which had the lowest concentrations across the sites with available data). The significance of this relationship was rather low $\left(R^{2}<0.40\right)$ for all but the roadside sites. This suggests that higher $\mathrm{H}_{2} \mathrm{SO}_{4}$ concentrations favour greater formation rates (i.e. more particles can be formed) rather than necessarily promoting nucleation itself because of the competing effect of condensation onto the pre-existing particle population.

The normalised gradients $a_{\mathrm{N}}^{*}$ were found to be more negative at the background sites compared to their respective roadside sites and less negative in the UK (where $\mathrm{SO}_{2}$ is in greater abundance) compared to the other sites with relatively significant relationships. Plotting the average $\mathrm{SO}_{2}$ concentrations with the normalised gradients $a_{\mathrm{N}}^{*}$ for the all sites (though not all had significant relationships), a positive relationship with relatively high $R^{2}$ (when the extreme values from Marylebone Road-UKRO are removed) is found, which might indicate that while increased concentrations are a negative factor in NPF event occurrence at a given site, in general the sites with higher $\mathrm{SO}_{2}$ concentrations on average present a higher frequency of NPF events (Fig. 7a and b). This appears to be in agreement with Dall'Osto et al. (2018), who discussed the variable role of $\mathrm{SO}_{2}$ depending on its concentrations. Similar findings for the effect of $\mathrm{SO}_{2}$ were also found in previous studies (Jung et al., 2006, 2008), relating particle acidity to NPF. Finally, no significant relationships were found for the values of $a_{J}^{*}$ as in most cases these relationships were rather weak.

\subsubsection{Nitrogen oxides or nitrogen dioxide $\left(\mathrm{NO}_{x}\right.$ or $\mathrm{NO}_{2}$ )}

$\mathrm{NO}_{x}$ and $\mathrm{NO}_{2}$ are directly associated with pollution, which can be a limiting factor for NPF events as it increases the 


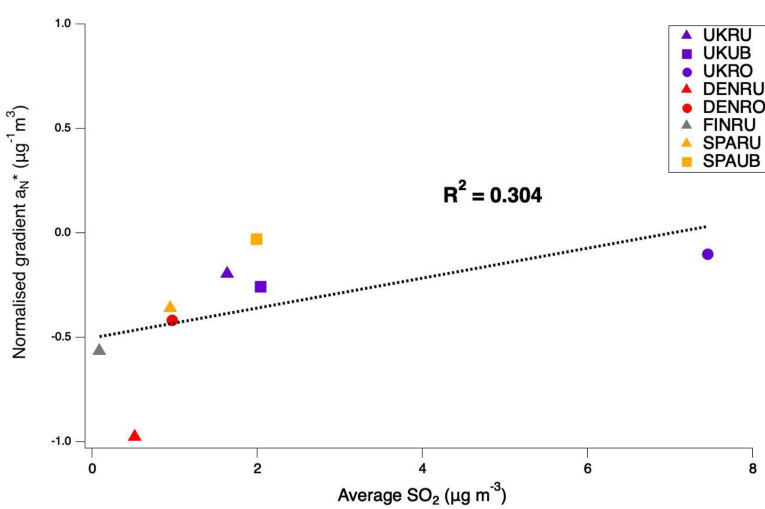

(a)

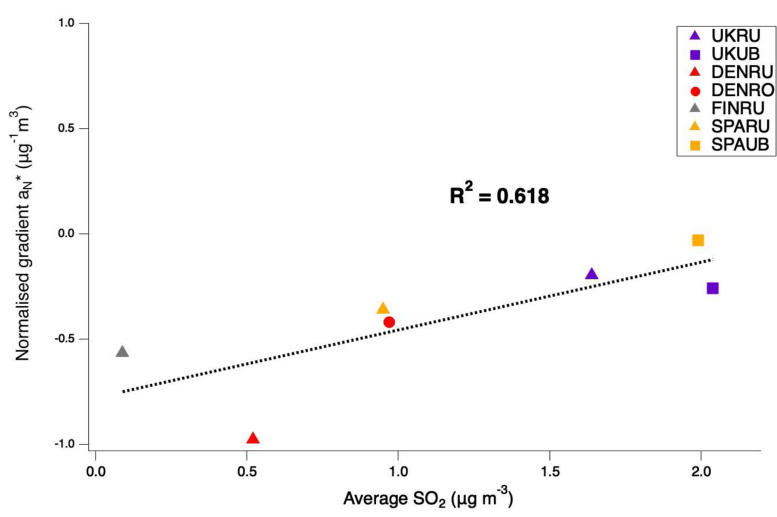

(b)

Figure 7. Relationship of average $\mathrm{SO}_{2}$ concentrations and normalised gradients $a_{\mathrm{N}}^{*}$ for the sites with available data (a) and for the sites with available data excluding UKRO (b).

CS and may suppress the events (An et al., 2015), though with the reduction of $\mathrm{SO}_{2}$ concentrations achieved the last couple of decades, there is a possibility for oxidation products of $\mathrm{NO}_{x}$ to become an important component for NPF (Wang et al., 2020). For almost all sites (apart from GRERU) with available data a negative relationship between the NPF frequency and $\mathrm{NO}_{x}$ concentrations (or $\mathrm{NO}_{2}$ depending on the available data) was found. Similarly, for all the sites but SPARU and GRERU, the correlations were relatively strong, with $R^{2}>0.43$. The rural background sites had a weaker relationship between the two variables compared to the urban sites, which is probably associated with them having rather low concentrations and variability of $\mathrm{NO}_{x}\left(\right.$ or $\left.\mathrm{NO}_{2}\right)$, making the variations of this factor less important. Growth rate had weaker correlations with $\mathrm{NO}_{x}$ and different trends between the sites, either being positive or negative. The variable effect of $\mathrm{NO}_{x}$ on particle growth, shifting highly oxygenated organic molecule (HOM) volatility, was previously discussed by Yan et al. (2020). While variability was found for the background sites, all roadside sites regardless of the strength of the relationship had a positive relationship between $\mathrm{NO}_{x}$ and the growth rate. This may indicate the different components associated with the growth process at each type of site which, as found in other studies, can be related to compounds associated with combustion processes that take place within the urban environment (Guo et al., 2020; S. Wang et al., 2017). The formation rate presents few cases of strong relationships, with variable trends (positive and negative). While much effort was made to isolate the effect of NPF events by taking a shorter timeframe before the event, the effect of local pollution is still included, especially at the urban sites (which probably explains the positive effect found).

The normalised gradients do not provide a significant result for the relationship of this variable with either the frequency of the events or the formation rate. The only noteworthy point is the more negative $a_{\mathrm{N}}^{*}$ at the rural background sites compared to the roadside sites in all the areas studied, which shows the increased importance of a clean environment for NPF events to occur in areas where condensable compounds are in lesser abundance, such as a rural environment. Additionally, negative gradients were found at all the roadside sites, which increases the confidence that the events extracted at the roadside sites are not pollution incidents but NPF events. However, it appears that traffic pollution favours higher particle growth rates, although the components responsible for this effect are unknown.

\subsubsection{Ozone $\left(\mathrm{O}_{3}\right)$}

Ozone is typically the result of atmospheric photochemistry and is itself a source of the hydroxyl radical through photolysis or ozonolysis of alkenes during both daytime and nighttime (Fenske et al., 2000). It might therefore be expected to act as an indicator of photochemical activity, which promotes the oxidation of $\mathrm{SO}_{2}$ and VOCs. Ozone concentrations may be directly related to the solar radiation intensity and the pollution levels in the area studied, and $\mathrm{O}_{3}$ is considered a positive factor in the occurrence of NPF events (Woo et al., 2001; Berndt et al., 2006). As with the solar radiation intensity, there is a strong relationship between $\mathrm{O}_{3}$ concentration and the frequency for NPF events. This positive relationship, which is in agreement with the higher concentrations of $\mathrm{O}_{3}$ found on NPF event days compared to average conditions for all sites in Bousiotis et al. $(2019,2020)$, was found to be stronger for the sites in northern Europe $\left(R^{2}>0.51\right)$, while it was not significant $\left(R^{2}<0.38\right)$ for the sites in southern Europe (Spanish sites and GRERU), possibly indicating that $\mathrm{O}_{3}$ is a less important factor at the southern sites. Specifically for the Spanish sites, which have the highest average concentrations of $\mathrm{O}_{3}$ with some extreme values (Querol et al., 2017), the relationship of $\mathrm{O}_{3}$ concentrations with the NPF frequency presents a unique trend (Fig. S8d), having a clear peak then a steady decline at both sites (though at different $\mathrm{O}_{3}$ concentrations), which is also responsible for the low correlations found (this trend seems to also occur at SPARU for the growth rate and to a lesser extent for the formation rate as well, though for different $\mathrm{O}_{3}$ concentration ranges - Fig. S8i and $n$ ). The specific variability found at the Spanish sites was also studied by Carnerero et al. (2019). For sites with 


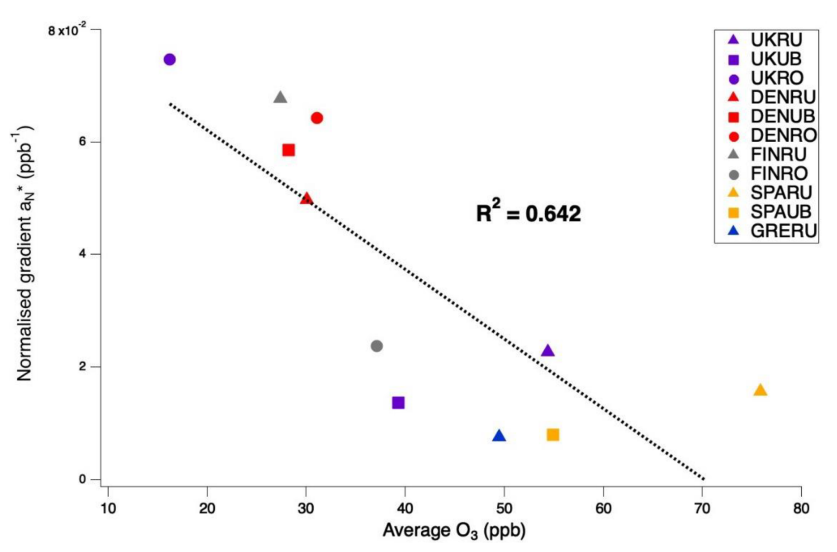

Figure 8. Relationship of average $\mathrm{O}_{3}$ concentrations and normalised gradients $a_{\mathrm{N}}^{*}$.

a marked seasonal variation in ozone, associations with NPF may be artefactual due to correlations with other variables such as temperature, $\mathrm{RH}$, and solar radiation intensity.

Unlike the solar radiation intensity, however, the growth rate presents a negative relationship at the sites where the relationship between these two variables was significant (UKRU, UKUB, DENUB, and FINRU), which might either be an indication of a polluted background that may have a negative effect on the growth of newly formed particles (though the trends found for $\mathrm{NO}_{x}$ indicate differently) or specific chemical processes which cannot be identified due to the lack of detailed chemical composition data. A significant relationship between $\mathrm{O}_{3}$ and the formation rate was only found for two sites (UKRO and DENRO, though the trends become a lot clearer if some values are removed from the extreme lower or higher end). This way, the relationships become strong but positive for some areas and negative for some others, without any clear trend (type or location of the site, $\mathrm{O}_{3}$ concentrations, etc.). No clear relationship between these two variables was found, as the sites with a strong relationship demonstrate both positive (DENRO) and negative (UKRO) relationships, and as a result no confident conclusions can be drawn.

As the correlations found were strong, the normalised gradients for NPF frequency, when plotted against the average concentrations of $\mathrm{O}_{3}$, present a negative correlation with relatively high $R^{2}(0.64)$, indicating that $\mathrm{O}_{3}$ is a more important factor in the occurrence of NPF events when in lower concentrations (Fig. 8). Finally, though with a low level of confidence for the southern sites, $a_{\mathrm{N}}^{*}$ was smaller at the southern sites compared to those in the north by up to 1 order of magnitude between FINRU (furthest north rural background) and GRERU (furthest south rural background).

\subsubsection{Organic compounds}

\section{Particulate organic carbon (OC)}

Organic carbon (OC) compounds in secondary aerosol typically enter particles via condensational processes, with a role that becomes increasingly important as the size of the particles becomes larger (Nieminen et al., 2010; Zhang et al., 2012; Shrivastava et al., 2017). Particulate OC, data for which are available in the present study, can be associated with pollution, especially in the urban environment. Only a few of the sites in the present study were found to have a relatively strong negative relationship $\left(R^{2}>0.50\right)$ of particulate OC with the NPF frequency (UKUB, UKRO, and DENRU). Regardless of the strength of this relationship, all other sites (apart from FINRU) had a negative relationship between these two variables as well, consistent with increased concentrations of particulate $\mathrm{OC}$ being associated with increased pollution, which elevates the CS, suppressing the occurrence of NPF events. The growth rate, on the other hand, was found to have a positive relationship $\left(R^{2}>0.40\right)$ for most of the sites. This relationship appeared to be stronger (higher $R^{2}$ ) at the roadside sites with available data compared to their respective rural background sites. The relationship between particulate $\mathrm{OC}$ and the growth rate was positive at all the sites with available data regardless of their significance, showing that, despite its effect on the occurrence of NPF events, it is still a favourable variable for the growth of particles. The formation rate was found to have a significant relationship with particulate $\mathrm{OC}$ concentrations at half of the sites with available data (UKUB, UKRO, DENRU, DENRO).

The normalised gradients for this variable did not present any noteworthy relationships with either the type of site or the concentrations of $\mathrm{OC}$ at a given site.

\section{Volatile organic compounds (VOCs)}

Many volatile organic compounds have been found to be associated with the NPF process. Benzene, toluene, ethylbenzene, $m$ - $p$-xylene, $o$-xylene, and trimethylbenzenes have been reported to be able to form highly oxygenated organic molecules (HOMs) in flow tubes (S. Wang et al., 2017; Molteni et al., 2018), which may act as contributors to particle nucleation and/or growth. Xylenes, and to a lesser extent trimethylbenzenes, are the most efficient at forming HOMs. Benzene and toluene are less efficient and will form more volatile HOMs. These HOMs may all be too volatile to form new particles, though this is not yet confirmed. Chamber studies involving $\mathrm{H}_{2} \mathrm{SO}_{4}$ and trimethylbenzene oxidation products were associated with high formation rates when measuring $J_{1.5}$ (Metzger et al., 2010). All these HOMs will be sufficiently involatile to contribute to particle growth. Those with a higher oxygen content or carbon number will be classed as low-volatility organic compounds (LVOCs), and if they dimerise, they will form extremely low-volatility or- 
ganic compounds (ELVOCs) (Bianchi et al., 2019). Monoterpenes can also form HOMs, which drive both formation (Ehn et al., 2014; Riccobono et al., 2014) and growth (Tröstl et al., 2016), while isoprene can act as a sink for the hydroxyl radical (Kiendler-Scharr et al., 2009) and is not as effective in $\mathrm{HOM}$ and secondary organic aerosol formation compared to monoterpenes (McFiggans et al., 2019).

Volatile organic compound data were available for three of the sites in this study (Table S2). Two of the sites with VOC data were from the rural background and the roadside site in the UK. Most of the compounds are associated with combustion sources and were found to have a negative relationship with NPF event occurrence at both sites, with high $R^{2}\left(R^{2}>0.50\right)$ in most cases. Additionally, isoprene, which may have either biogenic or anthropogenic sources (Wagner and Kuttler, 2014), was also found to have a negative relationship with NPF event occurrence at Marylebone Road-UKRO, though with low $R^{2}(0.07)$. This result is in line with the VOCs being strongly correlated with particulate OC (which presented a negative relationship with NPF event frequency, as discussed in Sect. 3.2.4) and with the CS (which also presented a negative relationship with NPF event frequency, as mentioned in Sect. 3.2.6), further associating these compounds with combustion emissions.

Growth rate was found to have a positive relationship with VOCs in almost all cases for both UK sites. A few exceptions were found (with only 1,3 butadiene having a relatively high $R^{2}$ ), which presented a negative relationship with the growth rate in rural Harwell-UKRU. Finally, the formation rate presented a different behaviour between the two sites. At UKRU, the relationship was unclear in most cases, with a group of VOCs presenting a negative relationship with the formation rate (ethane, ethene, propane, 1,3 butadiene, toluene, ethylbenzene, $o$-xylene, and 1,2,4 trimethylbenzene - with $R^{2}>0.40$ ); two VOCs presented a rather clear positive relationship with the formation rate (iso-pentane and 2methylbenzene), and the rest of the VOCs had an unclear relationship. At UKRO, however, VOCs presented a positive relationship with the formation rate (for particles of diameter $16 \mathrm{~nm}$ ). This is probably due to the fact that these VOCs are associated with pollution emissions (as mentioned earlier), and though a smaller time window was chosen to avoid including the effect of the morning rush hour traffic, this is very difficult in the traffic-polluted environment of Marylebone Road.

As Hyytiälä (FINRU) is a rural background site far from the direct effect of combustion emissions, different VOCs were measured, which mainly originate from biogenic sources rather than anthropogenic ones. The results were mixed and less clear compared to those from the UK sites (mainly due to the smaller dataset), and three groups were found depending on their relationship with NPF frequency. The first group, including acetonitrile, acetic acid, and methyl ethyl ketone (MEK), presented a slight positive relationship. The second group presented a negative relationship, with the VOCs in this group being monoterpenes, methacrolein, benzene, isoprene, and toluene (only the last two have $R^{2}>0.50$ ). Finally, the third group included VOCs that presented a peak and then a decline for higher concentrations, including methanol and acetone. Two groups of VOCs were found depending on their relationship with the growth rate. The ones with a positive relationship are methanol, acetonitrile, acetone, acetic acid, isoprene, methacrolein, monoterpenes, and toluene, while acetaldehyde, MEK, and benzene had a negative relationship, with relatively high $R^{2}$ in most cases. Finally, the results for the formation rate were unclear, with only a handful presenting weak $\left(R^{2}<0.21\right)$ positive (methanol, acetic acid, and benzene) or negative (MEK) relationships that do not appear to be significant. The normalised gradients cannot be used for VOCs as there are very few sites with available data.

\subsubsection{Sulfate $\left(\mathrm{SO}_{4}^{2-}\right)$}

Sulfate $\left(\mathrm{SO}_{4}^{2-}\right)$ is a major secondary constituent of aerosols. Secondary $\mathrm{SO}_{4}^{2-}$ aerosols largely arise from either gas-phase reaction between $\mathrm{SO}_{2}$ and $\mathrm{OH}$, or in the aqueous phase through the reaction of $\mathrm{SO}_{2}$ and $\mathrm{O}_{3}, \mathrm{H}_{2} \mathrm{O}_{2}$, or $\mathrm{NO}_{2}$ (Hidy, 1994). In environments where $\mathrm{SO}_{4}^{2-}$ chemistry is dominant (i.e. remote areas), $\mathrm{SO}_{4}^{2-}$ and ammonium (bi)sulfate $\left(\left(\mathrm{NH}_{4}\right)_{2} \mathrm{SO}_{4}\right.$ and $\left.\mathrm{NH}_{4} \mathrm{HSO}_{4}\right)$ particles are large relative contributors to aerosol mass, while this contribution is lower in environments where other emissions are also significant (i.e. urban areas where the secondary $\mathrm{NO}_{3}^{-}$relative contribution is a lot higher). While not well established, a possible relationship of $\mathrm{SO}_{4}^{2-}$-containing compounds and variables with NPF events was found in previous studies (Beddows et al., 2015; Minguillón et al., 2015; Z. Wang et al., 2017). In the present study, only a few sites had $\mathrm{SO}_{4}^{2-}$ data available for $\mathrm{PM}_{1}$ (FINRU), $\mathrm{PM}_{2.5}$ (Danish sites), or $\mathrm{PM}_{10}$ (rest of the sites). While these data cannot be considered to be directly associated with ultrafine particles, for two sites with available Aerosol Chemical Speciation Monitor (ACSM) data for ultrafine particles, the direct comparison between $\mathrm{SO}_{4}^{2-}$ aerosol in PM and in the range of particles of about $50 \mathrm{~nm}$ revealed very high correlations (results not included). For all the sites with available data the NPF frequency presented a negative relationship. The significance of this relationship was found to be relatively high $\left(R^{2}>0.50\right)$ only for background sites (apart from GERRU, which has rather low concentrations and probably different mechanisms for the NPF events). Similarly, the growth rate presented a significant relationship $\left(R^{2}>0.40\right)$ for the same background sites (apart from FINRU), though this relationship was found to be positive at all sites regardless of its significance. Finally, the formation rate did not present a clear trend as it was found to have both negative and positive relationships for different sites. This relationship was significant for only two rural sites 
(UKRU and DENRU), and as a result no conclusions can be reached.

The normalised gradients cannot be used for any analysis of sulfate as the measurements available are from different particle size ranges.

\subsubsection{Gaseous ammonia $\left(\mathrm{NH}_{3}\right)$}

Ammonia $\left(\mathrm{NH}_{3}\right)$ can be an important compound in the nucleation process according to the ternary theory (Kirkby et al., 2011; Napari et al., 2002). It was found that elevations in $\mathrm{NH}_{3}$ concentrations can lead to elevations in the NPF rate (Lehtipalo et al., 2018), and it was also found to be an important factor for NPF event occurrence even when stronger bases are present in high concentrations (Glasoe et al., 2015). No significant variation was found between event and nonevent days in a previous study in Harwell-UKRU (Bousiotis et al., 2019). Data for gaseous ammonia were only available for UKRU and presented a positive relationship with NPF frequency until reaching a peak point. A further increase in $\mathrm{NH}_{3}$ concentrations presented a decline with NPF frequency (Fig. S11a), which might be due to its association with increased pollution levels. It presented a clear positive relationship with both the growth rate (though it also appears to decline at high concentrations) and the formation rate, consistent with its well-established role in accelerating both of these processes (Kirkby et al. 2011; Stolzenburg et al., 2020).

\subsubsection{Condensation sink (CS)}

The CS is a measure of the rate at which molecules will condense onto pre-existing aerosols (Lehtinen et al., 2003). It is highly dependent on the number and size of the particles in the atmosphere, and as a result it is expected to be affected by both local emissions within the urban environment and the formation and growth of particles due to NPF events. As a result, for the specific metric a timeframe before the events are in full development was chosen (05:00 to 10:00 LT) to avoid including the effect of the NPF events and provide a picture of the atmospheric conditions that preceded the NPF events. With these data, the NPF frequency presented very strong relationships with the condensation sink. Two groups of sites were found: those which had a positive relationship and those with a negative relationship. In the first group are the sites in Germany and Greece, while all others had a negative relationship. This grouping follows the trend between the countries, the sites of which presented a greater or smaller CS on NPF event days according to the findings in Bousiotis et al. $(2019,2020)$ (having positive or negative gradients, respectively), though it is unknown what causes this behaviour (at the German sites and GREUB it may be associated with the very high formation rates on NPF event days). While the gradients from this analysis cannot be used for direct comparisons, a trend was found for which the gradients were more positive or negative at the rural sites compared to their re- spective roadside sites, which might indicate the greater importance of the variability of the CS at the rural sites for the occurrence of NPF events.

The growth rate was positively correlated with the CS for most of the sites, with relatively strong relationships $\left(R^{2}>0.40\right)$ for about half of them. As the CS is a metric of pre-existing particles, it is also associated with the level of pollution in a given area. The increased significance and gradient found at the rural sites probably indicate the importance of the enhanced presence of condensable compounds in a cleaner environment, which in many cases are associated with the moderate presence of pollution. The formation rate was also found to have a positive relationship with the CS. This relationship was more significant at the roadside sites in this study, a result which to some extent is biased by the presence of increased traffic emissions found in the timeframe chosen. While to an extent the increased presence of condensable compounds can be favourable for higher formation rates, this result should be considered with great caution.

The normalised gradients $a_{\mathrm{N}}^{*}$ followed a similar trend as those found with the initial analysis. These gradients were found to be more positive or negative, depending on the trend of the given area, at the rural sites compared to their roadside sites. The urban background sites did not always have a uniform behaviour (though in the UK, Denmark, and Finland these were between the rural site and the roadside site) due to their more diverse character compared to the other two types of sites.

\subsection{Association of the effect of the variables}

The Pearson correlation coefficients for the variables studied at each site are found in Table S1. The relatively strong relationship between the solar radiation intensity, temperature, and $\mathrm{O}_{3}$ found, in addition to their anticorrelation with the $\mathrm{RH}$, may lead to the conclusion that not all these factors play a role in NPF events, but their visible effect is the result of their relationship with each other. There is a similar case with the association of the $\mathrm{CS}$ and $\mathrm{NO}_{x}$ (or $\mathrm{NO}_{2}$ ), $\mathrm{OC}$, and $\mathrm{SO}_{2}$, especially at urban sites. However, the factors affect different outcomes differently; for example, the solar radiation intensity does not seem to be as important a factor for the growth rate as temperature, and $\mathrm{O}_{3}$ does not seem to be strongly associated with either the formation or the growth rate. This is further established by the fact that some of these variables do not correlate well at the southern sites but still appear to be associated with either the frequency of NPF events or the growth or nucleation rate. The effects of all of these factors have been demonstrated in both laboratory and atmospheric studies in the past and were discussed earlier in this paper. Through the analysis provided in the present study, the effect of each of these variables is further established, providing an association of each one of these variables with either the formation or the growth mechanism. However, RH does not seem to be a consistent factor in any mechanism, and it 
appears that its effect is dependent on location-specific conditions, although it was the variable with the most consistent relation with NPF event frequency at almost all sites.

\subsection{Relationship to a previous multi-station European study}

The findings of our study with respect to the background sites show many similarities to the conclusions drawn in a previous multi-station study in Europe by Dall'Osto et al. (2018) despite the two studies using several different sampling stations in addition to some in common. Both studies point towards the influence of variables such as solar radiation intensity and CS upon the occurrence of NPF events. The previous study suggested that different compounds participate in the growth of particles depending on the area considered. Thus, for northern and southern sites the growth of the particles is suggested to be driven mainly by organic compounds, while for the sites in central Europe sulfate plays a more important role. These findings are confirmed by the present study, as the growth rate was found to correlate better with organic compounds for the rural sites in Finland and Greece, while $\mathrm{SO}_{4}^{2-}$ presented a stronger relationship with the growth rate for the Danish and German sites (the latter presented high gradient values but low $R^{2}$ due to a decline at higher $\mathrm{SO}_{4}^{2-}$ concentrations, probably associated with NPF events being suppressed by increased pollution - Fig. S10i). The growth of the particles at the rural background site in the UK, characterised as "overlap" in the previous study, was found to be strongly associated with both organic compounds and sulfate, consistent with it being in the central group.

The seasonality of NPF events at northern sites was hard to explain in the previous study, and the possible effect of low temperature was considered. In the present study, the Finnish background sites presented a double-peak relationship of NPF frequency with temperature, with one of the peaks being below $0^{\circ}$. This might point to the possibility of different compounds driving the events for different temperature ranges and the increased nucleation rate of $\mathrm{H}_{2} \mathrm{SO}_{4}$ at lower temperatures (Kirkby et al., 2011; Yan et al., 2018), which makes the occurrence of NPF events more probable at lower temperatures in a region with low $\mathrm{SO}_{2}$ concentrations.

\section{Conclusions}

The present study attempts to explain the effect of several meteorological and atmospheric variables on the occurrence and development of NPF events by using a large-scale dataset. More than 85 site years of data from 16 sites from six countries in Europe were analysed for NPF events. A total of 1952 NPF events with consequent growth of newly formed particles were extracted, and with the use of binned linear regression, the relationship between three variables associated with NPF events (NPF event frequency, formation, and growth rate) and meteorological conditions as well as atmospheric composition was studied. Among the meteorological conditions, solar radiation intensity, temperature, and atmospheric pressure presented a positive relationship with the occurrence of NPF events at the majority of the sites (though exceptions were found as well, mostly in the southern sites), either promoting the formation or growth rate. $\mathrm{RH}$ presented a negative relationship with NPF event frequency, which in most cases was associated with it being a limiting factor on particle formation at higher average values. Wind speed, on the other hand, presented variable results, appearing to depend on the location of the sites rather than their type. This shows that while wind speed can be a factor in NPF event occurrence, the origin of the incoming air masses also plays a very important role. In most cases, meteorological conditions, such as temperature or $\mathrm{RH}$, appeared to be more important factors in NPF event occurrence at rural sites compared to urban sites, suggesting that NPF events are driven more by them at this type of site compared to urban environments and the more complex chemical interactions found there. Additionally, while some meteorological variables appeared to play a crucial role in the occurrence of NPF events, this role appears to become less important at higher values when a positive relation is found (or lower when a negative relation is found).

The results for the levels of atmospheric pollutants presented a more interesting picture, as most of these, which appear to be either directly or indirectly associated with the NPF process, were found to have negative relationships with NPF frequency. This is probably due to the fact that increased concentrations of such compounds are associated with more polluted conditions, which are a limiting factor in the occurrence of NPF events, as was found with the negative relationship between the CS and NPF frequency in most cases. Thus, $\mathrm{SO}_{2}, \mathrm{NO}_{x}$ (or $\mathrm{NO}_{2}$ ), particulate $\mathrm{OC}$, and $\mathrm{SO}_{4}^{2-}$ concentrations were negatively correlated with NPF frequency in most cases. Average $\mathrm{SO}_{2}$ concentrations appeared to correlate positively with the normalised NPF event frequency gradients with a relatively significant correlation, indicating that while increasing concentrations have a negative impact on the occurrence of NPF events at a given site, in general sites with higher $\mathrm{SO}_{2}$ concentrations have a higher frequency of NPF events. Conversely, these compounds in many cases had a positive relationship (though not always with high significance) with the other variables considered. Thus, particulate OC (and VOCs when data were available) and $\mathrm{SO}_{4}^{2-}$ consistently had a positive relationship with the growth rate, while $\mathrm{SO}_{2}$ was positively associated with both the formation and growth rate in most cases. Finally, $\mathrm{O}_{3}$ was positively correlated with NPF event frequency at all sites in this study, though it presented variable results with the other two variables. As with some meteorological conditions it was found that at sites with increased concentrations of $\mathrm{O}_{3}$, its importance as a factor was decreased, which to some extent can be 
related to the high $\mathrm{CS}$ associated with peak summer $\mathrm{O}_{3}$ days in southern Europe.

It should be noted that the variables considered are in many cases inter-related (e.g. temperature and $\mathrm{RH}$ ), and this considerably complicates the interpretation in terms of causal factors. Large datasets are very useful in providing more uniform results by removing the possible bias of short period extremities, which may lead to wrong assumptions. This study, apart from providing insights into the effect of a number of variables on the occurrence and development of NPF events in atmospheric conditions across Europe, also shows the differences that climatic, land use, and atmospheric composition variations cause in those effects. Such variations are probably the cause of the differences found among previous studies. Following from this, the importance of a highresolution measurement network, both spatially and temporally, is underlined, as it can help in elucidating the mechanisms of new particle formation in the real atmosphere.

Data availability. Data supporting this publication are openly available from the UBIRA eData repository at https://doi.org/10.25500/edata.bham.00000491 (Harrison and Bousiotis, 2021).

Supplement. The supplement related to this article is available online at: https://doi.org/10.5194/acp-21-3345-2021-supplement.

Author contributions. The study was conceived and planned by $\mathrm{RMH}$, who also contributed to the final paper, and DB, who also carried out the analysis and prepared the first draft of the paper. AM, JKN, CN, JVN, HP, NP, AA, GK, SV, and KE provided the data for the analysis. JB provided help with analysis of the data. FP provided advice on the analysis. MD' $\mathrm{O}, \mathrm{XQ}$, and TP contributed to the final paper.

Competing interests. The authors declare that they have no conflict of interest.

Acknowledgements. This work was supported by the National Centre for Atmospheric Science funded by the UK Natural Environment Research Council (R8/H12/83/011).

Financial support. This research has been supported by the Natural Environment Research Council (grant no. R8/H12/83/011).

Review statement. This paper was edited by Gordon McFiggans and reviewed by two anonymous referees.

\section{References}

Aalto, P., Hämeri, K., Becker, E. D. O., Weber, R., Salm, J., Mäkelä, J. M., Hoell, C., O’Dowd, C. D., Karlsson, H., Hansson, H., Väkevä, M., Koponen, I. K., Buzorius, G., and Kulmala, M.: Physical characterization of aerosol particles during nucleation events, Tellus B, 53, 344-358, https://doi.org/10.3402/tellusb.v53i4.17127, 2001.

Alam, A., Shi, J. P., and Harrison, R. M.: Observations of new particle formation in urban air, J. Geophys. Res.-Atmos., 108, 40934107, https://doi.org/10.1029/2001JD001417, 2003.

An, J., Wang, H., Shen, L., Zhu, B., Zou, J., Gao, J., and Kang, H.: Characteristics of new particle formation events in Nanjing, China: Effect of water-soluble ions, Atmos. Environ., 108, 3240, https://doi.org/10.1016/j.atmosenv.2015.01.038, 2015.

Bae, M.-S., Schwab, J. J., Hogrefe, O., Frank, B. P., Lala, G. G., and Demerjian, K. L.: Characteristics of size distributions at urban and rural locations in New York, Atmos. Chem. Phys., 10, 45214535, https://doi.org/10.5194/acp-10-4521-2010, 2010.

Beddows, D. C. S. and Harrison, R. M.: Receptor modelling of both particle composition and size distribution from a background site in London, UK - a two-step approach, Atmos. Chem. Phys., 19, 4863-4876, https://doi.org/10.5194/acp-19-4863-2019, 2019.

Beddows, D. C. S., Harrison, R. M., Green, D. C., and Fuller, G. W.: Receptor modelling of both particle composition and size distribution from a background site in London, UK, Atmos. Chem. Phys., 15, 10107-10125, https://doi.org/10.5194/acp-15-101072015, 2015.

Berland, K., Rose, C., Pey, J., Culot, A., Freney, E., Kalivitis, N., Kouvarakis, G., Cerro, J. C., Mallet, M., Sartelet, K., Beckmann, M., Bourriane, T., Roberts, G., Marchand, N., Mihalopoulos, N., and Sellegri, K.: Spatial extent of new particle formation events over the Mediterranean Basin from multiple ground-based and airborne measurements, Atmos. Chem. Phys., 17, 9567-9583, https://doi.org/10.5194/acp-17-9567-2017, 2017.

Berndt, T., Böge, O., and Stratmann, F.: Formation of atmospheric $\mathrm{H}_{2} \mathrm{SO}_{4} \mathrm{H}_{2} \mathrm{O}$ particles in the absence of organics: A laboratory study, Geophys. Res. Lett., 33, 2-6, https://doi.org/10.1029/2006GL026660, 2006.

Bianchi, F., Kurtén, T., Riva, M., Mohr, C., Rissanen, M. P., Roldin, P., Berndt, T., Crounse, J. D., Wennberg, P. O., Mentel, T. F., Wildt, J., Junninen, H., Jokinen, T., Kulmala, M., Worsnop, D. R., Thornton, J. A., Donahue, N., Kjaergaard, H. G., and Ehn, M.: Highly oxygenated organic molecules (HOM) from gas-phase autoxidation involving peroxy radicals: A key contributor to atmospheric aerosol, Chem. Rev., 119, 3472-3509, https://doi.org/10.1021/acs.chemrev.8b00395, 2019.

Bigi, A. and Harrison, R. M.: Analysis of the air pollution climate at a central urban background site, Atmos. Environ., 44, 20042012, https://doi.org/10.1016/j.atmosenv.2010.02.028, 2010.

Birmili, W., Weinhold, K., Rasch, F., Sonntag, A., Sun, J., Merkel, M., Wiedensohler, A., Bastian, S., Schladitz, A., Löschau, G., Cyrys, J., Pitz, M., Gu, J., Kusch, T., Flentje, H., Quass, U., Kaminski, H., Kuhlbusch, T. A. J., Meinhardt, F., Schwerin, A., Bath, O., Ries, L., Gerwig, H., Wirtz, K., and Fiebig, M.: Longterm observations of tropospheric particle number size distributions and equivalent black carbon mass concentrations in the German Ultrafine Aerosol Network (GUAN), Earth Syst. Sci. Data, 8, 355-382, https://doi.org/10.5194/essd-8-355-2016, 2016. 
Bousiotis, D., Dall'Osto, M., Beddows, D. C. S., Pope, F. D., and Harrison, R. M.: Analysis of new particle formation (NPF) events at nearby rural, urban background and urban roadside sites, Atmos. Chem. Phys., 19, 5679-5694, https://doi.org/10.5194/acp19-5679-2019, 2019.

Bousiotis, D., Pope, F. D., Beddows, D. C., Dall'Osto, M., Massling, A., Nøjgaard, J. K., Nordstrøm, C., Niemi, J. V., Portin, H., Petäjä, T., Perez, N., Alastuey, A., Querol, X., Kouvarakis, G., Vratolis, S., Eleftheriadis, K., Wiedensohler, A., Weinhold, K., Merkel, M., Tuch, T., and Harrison, R. M.: An Analysis of New Particle Formation (NPF) at Thirteen European Sites, Atmos. Chem. Phys. Discuss. [preprint], https://doi.org/10.5194/acp-2020-414, in review, 2020.

Brines, M., Dall'Osto, M., Beddows, D. C. S., Harrison, R. M., Gómez-Moreno, F., Núñez, L., Artíñano, B., Costabile, F., Gobbi, G. P., Salimi, F., Morawska, L., Sioutas, C., and Querol, X.: Traffic and nucleation events as main sources of ultrafine particles in high-insolation developed world cities, Atmos. Chem. Phys., 15, 5929-5945, https://doi.org/10.5194/acp15-5929-2015, 2015.

Carnerero, C., Pérez, N., Petäjä, T., Laurila, T. M., Ahonen, L. R., Kontkanen, J., Ahn, K. H., Alastuey, A., and Querol, X.: Relating high ozone, ultrafine particles, and new particle formation episodes using cluster analysis, Atmos. Environ., 4, 100051, https://doi.org/10.1016/j.aeaoa.2019.100051, 2019.

Charron, A. and Harrison, R. M.: Primary particle formation from vehicle emissions during exhaust dilution in the roadside atmosphere, Atmos. Environ., 37, 4109-4119, https://doi.org/10.1016/S1352-2310(03)00510-7, 2003.

Charron, A., Birmili, W., and Harrison, R. M.: Fingerprinting particle origins according to their size distribution at a UK rural site, J. Geophys. Res.-Atmos., 113, D07202, https://doi.org/10.1029/2007JD008562, 2008.

Charron, A., Degrendele, C., Laongsri, B., and Harrison, R. M.: Receptor modelling of secondary and carbonaceous particulate matter at a southern UK site, Atmos. Chem. Phys., 13, 1879-1894, https://doi.org/10.5194/acp-13-1879-2013, 2013.

Cheung, H. C., Chou, C. C.-K., Huang, W.-R., and Tsai, C.Y.: Characterization of ultrafine particle number concentration and new particle formation in an urban environment of Taipei, Taiwan, Atmos. Chem. Phys., 13, 8935-8946, https://doi.org/10.5194/acp-13-8935-2013, 2013.

Chu, B., Kerminen, V.-M., Bianchi, F., Yan, C., Petäjä, T., and Kulmala, M.: Atmospheric new particle formation in China, Atmos. Chem. Phys., 19, 115-138, https://doi.org/10.5194/acp-19-1152019, 2019.

Dada, L., Paasonen, P., Nieminen, T., Buenrostro Mazon, S., Kontkanen, J., Peräkylä, O., Lehtipalo, K., Hussein, T., Petäjä, T., Kerminen, V.-M., Bäck, J., and Kulmala, M.: Long-term analysis of clear-sky new particle formation events and nonevents in Hyytiälä, Atmos. Chem. Phys., 17, 6227-6241, https://doi.org/10.5194/acp-17-6227-2017, 2017.

Dai, L., Wang, H., Zhou, L., An, J., Tang, L., Lu, C., Yan, W., Liu, R., Kong, S., Chen, M., Lee, S., and Yu, H.: Regional and local new particle formation events observed in the Yangtze River Delta region, China, J. Geophys. Res., 122, 2389-2402, https://doi.org/10.1002/2016JD026030, 2017.

Dall'Osto, M., Beddows, D. C. S., Pey, J., Rodriguez, S., Alastuey, A., Harrison, R. M., and Querol, X.: Urban aerosol size distributions over the Mediterranean city of Barcelona, NE Spain, Atmos. Chem. Phys., 12, 10693-10707, https://doi.org/10.5194/acp-12-10693-2012, 2012.

Dall'Osto, M., Querol, X., Alastuey, A., O’Dowd, C., Harrison, R. M., Wenger, J., and Gómez-Moreno, F. J.: On the spatial distribution and evolution of ultrafine particles in Barcelona, Atmos. Chem. Phys., 13, 741-759, https://doi.org/10.5194/acp-13-7412013, 2013.

Dall'Osto, M., Beddows, D. C. S., Asmi, A., Poulain, L., Hao, L., Freney, E., Allan, J. D., Canagaratna, M., Crippa, M., Bianchi, F., De Leeuw, G., Eriksson, A., Swietlicki, E., Hansson, H. C., Henzing, J. S., Granier, C., Zemankova, K., Laj, P., Onasch, T., Prevot, A., Putaud, J. P., Sellegri, K., Vidal, M., Virtanen, A., Simo, R., Worsnop, D., O'Dowd, C., Kulmala, M., and Harrison, R. M.: Novel insights on new particle formation derived from a pan-european observing system, Sci. Rep.-UK, 8, 1482, https://doi.org/10.1038/s41598-017-17343-9, 2018.

Dal Maso, M., Kulmala, M., Riipinen, I., Wagner, R., Hussein, T., Aalto, P. P., and Lehtinen, K. E. J.: Formation and growth of fresh atmospheric aerosols: Eight years of aerosol size distribution data from SMEAR II, Hyytiälä, Finland, Boreal Environ. Res., 10, 323-336, https://doi.org/10.1016/j.ijpharm.2012.03.044, 2005.

Ehn, M., Thornton, J. A., Kleist, E., Sipilä, M., Junninen, H., Pullinen, I., Springer, M., Rubach, F., Tillmann, R., Lee, B., Lopez-Hilfiker, F., Andres, S., Acir, I. H., Rissanen, M., Jokinen, T., Schobesberger, S., Kangasluoma, J., Kontkanen, J., Nieminen, T., Kurtén, T., Nielsen, L. B., Jørgensen, S., Kjaergaard, H. G., Canagaratna, M., Maso, M. D., Berndt, T., Petäjä, T., Wahner, A., Kerminen, V.-M., Kulmala, M., Worsnop, D. R., Wildt, J., and Mentel, T. F.: A large source of lowvolatility secondary organic aerosol, Nature, 506, 476-479, https://doi.org/10.1038/nature13032, 2014.

Fenske, J. D., Hasson, A. S., Paulson, S. E., Kuwata, K. T., Ho, A., and Houk, K. N.: The Pressure Dependence of the OH Radical Yield from Ozone Alkene Reactions, J. Phys. Chem. A, 104, 7821, https://doi.org/10.1021/jp001100u, 2000.

Fuchs, N. A. and Sutugin, A. G.: Highly Dispersed Aerosols, Foreign Sci. Technol. Center, 1-86, 1971.

Glasoe, W. A., Volz, K., Panta, B., Freshour, N., Bachman, 100 R., Hanson, D. R., McMurry, P. H., and Jen, C.: Sulfuric acid nucleation: An experimental study of the effect of seven bases, J. Geophys. Res.-Atmos., 120, 1933-1950, https://doi.org/10.1002/2014JD022730, 2015.

Größ, J., Hamed, A., Sonntag, A., Spindler, G., Manninen, H. E., Nieminen, T., Kulmala, M., Hõrrak, U., Plass-Dülmer, C., Wiedensohler, A., and Birmili, W.: Atmospheric new particle formation at the research station Melpitz, Germany: connection with gaseous precursors and meteorological parameters, Atmos. Chem. Phys., 18, 1835-1861, https://doi.org/10.5194/acp18-1835-2018, 2018.

Guo, S., Hu, M., Peng, J., Wu, Z., Zamora, M. L., Shang, D., Du, Z., Zheng, J., Fang, X., Tang, R., Wu, Y., Zeng, L., Shuai, S., Zhang, W., Wang, Y., Ji, Y., Li, Y., Zhang, A. L., Wang, W., Zhang, F., Zhao, J., Gong, X., Wang, C., Molina, M. J., and Zhang, R.: Remarkable nucleation and growth of ultrafine particles from vehicular exhaust, P. Natl. Acad. Sci. USA, 117, 34273432, https://doi.org/10.1073/pnas.1916366117, 2020. 
Hallar, A. G., Petersen, R., McCubbin, I. B., Lowenthal, D., Lee, S., Andrews, E., and Yu, F.: Climatology of new particle formation and corresponding precursors at storm peak laboratory, Aerosol Air Qual. Res., 16, 816-826, https://doi.org/10.4209/aaqr.2015.05.0341, 2016.

Hamed, A., Korhonen, H., Sihto, S. L., Joutsensaari, J., Jrvinen, H., Petäjä, T., Arnold, F., Nieminen, T., Kulmala, M., Smith, J. N., Lehtinen, K. E. J., and Laaksonen, A.: The role of relative humidity in continental new particle formation, J. Geophys. Res.Atmos., 116, D03202, https://doi.org/10.1029/2010JD014186, 2011.

Harrison, R. M.: Urban atmospheric chemistry: A very special case for study, npj Clim. Atmos. Sci., 1, 20175, https://doi.org/10.1038/s41612-017-0010-8, 2017.

Harrison, R. M. and Bousiotis, D.: Research data supporting "The Effect of Meteorological Conditions and Atmospheric Composition in the Occurrence and Development of New Particle Formation (NPF) Events in Europe", University of Birmingham, https://doi.org/10.25500/edata.bham.00000491, 2021.

Henschel, H., Kurtén, T., and Vehkamäki, H.: Computational study on the effect of hydration on new particle formation in the sulfuric acid/ammonia and sulfuric acid/dimethylamine systems, J. Phys. Chem. A, 120, 1886-1896, 2016.

Hidy, G. M.: Atmospheric sulfur and nitrogen oxides, Academic Press, San Diego, USA, 447 pp., ISBN 9781483288666, 1994.

Hietikko, R., Kuuluvainen, H., Harrison, R. M., Portin, H., Timonen, H., Niemi, J. V., and Rönkkö, T.: Diurnal variation of nanocluster aerosol concentrations and emission factors in a street canyon, Atmos. Environ., 189, 98-106, https://doi.org/10.1016/j.atmosenv.2018.06.031, 2018.

Iida, K., Stolzenburg, M. R., McMurry, P. H., and Smith, J. N.: Estimating nanoparticle growth rates from size-dependent charged fractions: Analysis of new particle formation events in Mexico City, J. Geophys. Res.-Atmos., 113, D05207, https://doi.org/10.1029/2007JD009260, 2008.

Järvi, L., Hannuniemi, H., Hussein, T., Junninen, H., Aalto, P., Hillamo, R., Mäkelä, T., Keronen, P., and Siivola, E.: The urban measurement station SMEAR III: Continuous monitoring of air pollution and surface - atmosphere interactions in Helsinki, Finland, Boreal Environ. Res., 14, 86-109, 2009.

Jayaratne, R., Pushpawela, B., He, C., Li, H., Gao, J., Chai, F., and Morawska, L.: Observations of particles at their formation sizes in Beijing, China, Atmos. Chem. Phys., 17, 8825-8835, https://doi.org/10.5194/acp-17-8825-2017, 2017.

Jeong, C.-H., Evans, G. J., McGuire, M. L., Chang, R. Y.-W., Abbatt, J. P. D., Zeromskiene, K., Mozurkewich, M., Li, S.M., and Leaitch, W. R.: Particle formation and growth at five rural and urban sites, Atmos. Chem. Phys., 10, 7979-7995, https://doi.org/10.5194/acp-10-7979-2010, 2010.

Jung, J., Adams P. J., and Pandis, S. N.: Simulating the size distribution and chemical composition of ultrafine particles during nucleation events, Atmos. Environ., 40, 2248-2259, https://doi.org/10.1016/j.atmosenv.2005.09.082, 2006.

Jung, J. G., Pandis, S. N., and Adams, P. J.: Evaluation of nucleation theories in a sulfur-rich environment, Aerosol Sci. Tech., 42, 495-504, https://doi.org/10.1080/02786820802187085, 2008.

Kalivitis, N., Kerminen, V.-M., Kouvarakis, G., Stavroulas, I., Tzitzikalaki, E., Kalkavouras, P., Daskalakis, N., Myriokefalitakis, S., Bougiatioti, A., Manninen, H. E., Roldin, P., Petäjä,
T., Boy, M., Kulmala, M., Kanakidou, M., and Mihalopoulos, N.: Formation and growth of atmospheric nanoparticles in the eastern Mediterranean: results from long-term measurements and process simulations, Atmos. Chem. Phys., 19, 2671-2686, https://doi.org/10.5194/acp-19-2671-2019, 2019.

Kalkavouras, P., Bossioli, E., Bezantakos, S., Bougiatioti, A., Kalivitis, N., Stavroulas, I., Kouvarakis, G., Protonotariou, A. P., Dandou, A., Biskos, G., Mihalopoulos, N., Nenes, A., and Tombrou, M.: New particle formation in the southern Aegean Sea during the Etesians: importance for $\mathrm{CCN}$ production and cloud droplet number, Atmos. Chem. Phys., 17, 175-192, https://doi.org/10.5194/acp-17-175-2017, 2017.

Kerminen, V.-M., Pirjola, L., and Kulmala, M.: How significantly does coagulational scavenging limit atmospheric particle production?, J. Geophys. Res.-Atmos., 106, 24119-24125, https://doi.org/10.1029/2001JD000322, 2001.

Kerminen, V.-M., Lehtinen, K. E. J., Anttila, T., Kulmala, M., Lehtinen, K. E. J., Anttila, T., and Kulmala, M.: Dynamics of atmospheric nucleation mode particles: a timescale analysis, Tellus B, 56, 135-146, https://doi.org/10.3402/tellusb.v56i2.16411, 2004.

Kerminen, V.-M., Kulmala, M., Worsnop, D. R., Wildt, J., and Mentel, T. F.: A large source of low-volatility secondary organic aerosol, Nature, 506, 476-479, https://doi.org/10.1038/nature13032, 2014.

Kerminen, V.-M., Chen, X., Vakkari, V., Petäjä, T., Kulmala, M., and Bianchi, F.: Atmospheric new particle formation and growth: review of field observations, Environ. Res. Lett., 13, 103003, https://doi.org/10.1088/1748-9326/aadf3c, 2018.

Ketzel, M., Wåhlin, P., Kristensson, A., Swietlicki, E., Berkowicz, R., Nielsen, O. J., and Palmgren, F.: Particle size distribution and particle mass measurements at urban,near-city and rural level in the Copenhagen area and Southern Sweden, Atmos. Chem. Phys., 4, 281-292, https://doi.org/10.5194/acp-4281-2004, 2004.

Kiendler-Scharr, A., Wildt, J., Dal Maso, M., Hohaus, T., Kleist, E., Mentel, T. F., Tillmann, R., Uerlings, R., Schurr, U., and Wahner, A.: New particle formation in forests inhibited by isoprene emissions, Nature, 461, 381-384, https://doi.org/10.1038/nature08292, 2009.

Kim, K. H., Kabir, E., and Kabir, S.: A review on the human health impact of airborne particulate matter, Environ. Int., 74, 136-143, https://doi.org/10.1016/j.envint.2014.10.005, 2015.

Kirkby, J., Curtius, J., Almeida, J., Dunne, E., Duplissy, J., Ehrhart, S., Franchin, A., Gagné, S., Ickes, L., Kürten, A., Kupc, A., Metzger, A., Riccobono, F., Rondo, L., Schobesberger, S., Tsagkogeorgas, G., Wimmer, D., Amorim, A., Bianchi, F., Breitenlechner, M., David, A., Dommen, J., Downard, A., Ehn, M., Flagan, R. C., Haider, S., Hansel, A., Hauser, D., Jud, W., Junninen, H., Kreissl, F., Kvashin, A., Laaksonen, A., Lehtipalo, K., Lima, J., Lovejoy, E. R., Makhmutov, V., Mathot, S., Mikkilä, J., Minginette, P., Mogo, S., Nieminen, T., Onnela, A., Pereira, P., Petäjä, T., Schnitzhofer, R., Seinfeld, J. H., Sipilä, M., Stozhkov, Y., Stratmann, F., Tomé, A., Vanhanen, J., Viisanen, Y., Vrtala, A., Wagner, P. E., Walther, H., Weingartner, E., Wex, H., Winkler, P. M., Carslaw, K. S., Worsnop, D. R., Baltensperger, U., and Kulmala, M.: Role of sulphuric acid, ammonia and galactic cosmic rays in atmospheric aerosol nucleation, Nature, 476, 429-435, https://doi.org/10.1038/nature10343, 2011. 
Korhonen, P., Kulmala, M., Laaksonen, A., Viisanen, Y., Mcgraw, R., and Seinfeld, J. H.: Ternary nucleation of $\mathrm{H}_{2} \mathrm{SO}_{4}, \mathrm{NH}_{3}$ and $\mathrm{H}_{2} \mathrm{O}$ in the atmosphere, J. Geophys. Res., 104, 26349-26353, 1999.

Kulmala, M. and Kerminen, V. M.: On the formation and growth of atmospheric nanoparticles, Atmos. Res., 90, 132-150, https://doi.org/10.1016/j.atmosres.2008.01.005, 2008.

Kulmala, M., Dal Maso, M., Mäkelä, J. M., Pirjola, L., Väkevä, M., Aalto, P., Miikkulainen, P., Hämeri, K., and O'Dowd, C. D.: On the formation, growth and composition of nucleation mode particles, Tellus B, 53, 479-490, https://doi.org/10.3402/tellusb.v53i4.16622, 2001.

Kulmala, M., Petäjä, T., Mönkkönen, P., Koponen, I. K., Dal Maso, M., Aalto, P. P., Lehtinen, K. E. J., and Kerminen, V.-M.: On the growth of nucleation mode particles: source rates of condensable vapor in polluted and clean environments, Atmos. Chem. Phys., 5, 409-416, https://doi.org/10.5194/acp-5-409-2005, 2005.

Kulmala, M., Petäjä, T., Nieminen, T., Sipilä, M., Manninen, H. E., Lehtipalo, K., Dal Maso, M., Aalto, P. P., Junninen, H., Paasonen, P., Riipinen, I., Lehtinen, K. E. J., Laaksonen, A., and Kerminen, V. M.: Measurement of the nucleation of atmospheric aerosol particles, Nat. Protoc., 7, 1651-1667, https://doi.org/10.1038/nprot.2012.091, 2012.

Kulmala, M., Kerminen, V.-M., Petäjä, T., Ding, A. J., and Wang, L.: Atmospheric gas-to-particle conversion: Why NPF events are observed in megacities?, Faraday Discuss., 200, 271-288, https://doi.org/10.1039/c6fd00257a, 2017.

Kürten, A., Bergen, A., Heinritzi, M., Leiminger, M., Lorenz, V., Piel, F., Simon, M., Sitals, R., Wagner, A. C., and Curtius, J.: Observation of new particle formation and measurement of sulfuric acid, ammonia, amines and highly oxidized organic molecules at a rural site in central Germany, Atmos. Chem. Phys., 16, 1279312813, https://doi.org/10.5194/acp-16-12793-2016, 2016.

Kürten, A., Li, C., Bianchi, F., Curtius, J., Dias, A., Donahue, N. M., Duplissy, J., Flagan, R. C., Hakala, J., Jokinen, T., Kirkby, J., Kulmala, M., Laaksonen, A., Lehtipalo, K., Makhmutov, V., Onnela, A., Rissanen, M. P., Simon, M., Sipilä, M., Stozhkov, Y., Tröstl, J., Ye, P., and McMurry, P. H.: New particle formation in the sulfuric acid-dimethylamine-water system: reevaluation of CLOUD chamber measurements and comparison to an aerosol nucleation and growth model, Atmos. Chem. Phys., 18, 845-863, https://doi.org/10.5194/acp-18-845-2018, 2018.

Lee, S.-H. H., Uin, J., Guenther, A. B., de Gouw, J. A., Yu, F., Nadykto, A. B., Herb, J., Ng, N. L., Koss, A., Brune, W. H., Baumann, K., Kanawade, V. P., Keutsch, F. N., Nenes, A., Olsen, K., Goldstein, A., and Ouyang, Q.: Isoprene suppression of new particle formation: Potential mechanisms and implications, J. Geophys. Res.-Atmos., 121, 14621-14635, https://doi.org/10.1002/2016JD024844, 2016.

Lehtinen, K. E. J., Korhonen, H., Dal Maso, M., and Kulmala, M.: On the concept of condensation sink diameter, Boreal Environ. Res., 8, 405-411, 2003.

Lehtipalo, K., Yan, C., Dada, L., Bianchi, F., Xiao, M., Wagner, R., Stolzenburg, D., Ahonen, L. R., Amorim, A., Baccarini, A., Bauer, P. S., Baumgartner, B., Bergen, A., Bernhammer, A., Breitenlechner, M., Brilke, S., Buchholz, A., Mazon, S. B., Chen, D., Chen, X., Dias, A., Dommen, J., Draper, D. C., Duplissy, J., Ehn, M., Finkenzeller, H., Fischer, L., Frege, C., Fuchs, C., Garmash, O., Gordon, H., Hakala, J., He, X., Heikkinen, L., Hein- ritzi, M., Helm, J. C., Hofbauer, V., Hoyle, C. R., Jokinen, T., Ojdanic, A., Onnela, A., Passananti, M., Petäjä, T., Piel, F., Sarnela, N., Schallhart, S., Schuchmann, S., Sengupta, K. and Simon, M.: Multicomponent new particle formation from sulfuric acid, ammonia, and biogenic vapors, Sci. Adv., 4, eaau5363, https://doi.org/10.1126/sciadv.aau5363, 2018.

Li, X., Chee, S., Hao, J., Abbatt, J. P. D., Jiang, J., and Smith, J. N.: Relative humidity effect on the formation of highly oxidized molecules and new particles during monoterpene oxidation, Atmos. Chem. Phys., 19, 1555-1570, https://doi.org/10.5194/acp19-1555-2019, 2019.

Makkonen, R., Asmi, A., Kerminen, V.-M., Boy, M., Arneth, A., Hari, P., and Kulmala, M.: Air pollution control and decreasing new particle formation lead to strong climate warming, Atmos. Chem. Phys., 12, 1515-1524, https://doi.org/10.5194/acp12-1515-2012, 2012.

McFiggans, G., Mentel, T. F., Wildt, J., Pullinen, I., Kang, S., Kleist, E., Schmitt, S., Springer, M., Tillmann, R., Wu, C., Zhao, D., Hallquist, M., Faxon, C., Le Breton, M., Hallquist, Å. M., Simpson, D., Bergström, R., Jenkin, M. E., Ehn, M., Thornton, J. A., Alfarra, M. R., Bannan, T. J., Percival, C. J., Priestley, M., Topping, D., and Kiendler-Scharr, A.: Secondary organic aerosol reduced by mixture of atmospheric vapours, Nature, 565, 587-593, https://doi.org/10.1038/s41586-018-0871-y, 2019.

Merikanto, J., Spracklen, D. V., Mann, G. W., Pickering, S. J., and Carslaw, K. S.: Impact of nucleation on global CCN, Atmos. Chem. Phys., 9, 8601-8616, https://doi.org/10.5194/acp-9-86012009, 2009.

Metzger, A., Verheggen, B., Dommen, J., Duplissy, J., Prevot, A. S. H., Weingartner, E., Riipinen, I., Kulmala, M., Spracklen, D. V., Carslaw, K. S., and Baltensperger, U.: Evidence for the role of organics in aerosol particle formation under atmospheric conditions, P. Natl. Acad. Sci. USA, 107, 6646-6651, https://doi.org/10.1073/pnas.0911330107, 2010.

Minguillón, M. C., Brines, M., Pérez, N., Reche, C., Pandolfi, M., Fonseca, A. S., Amato, F., Alastuey, A., Lyasota, A., Codina, B., Lee, H. K., Eun, H. R., Ahn, K. H., and Querol, X.: New particle formation at ground level and in the vertical column over the Barcelona area, Atmos. Res., 164-165, 118-130, https://doi.org/10.1016/j.atmosres.2015.05.003, 2015.

Mirabel, P. and Katz, J. L.: Binary homogeneous nucleation as a mechanism for the formation of aerosols, J. Chem. Phys., 60, 1138-1144, https://doi.org/10.1063/1.1681124, 1974.

Mølgaard, B., Birmili, W., Clifford, S., Massling, A., Eleftheriadis, K., Norman, M., Vratolis, S., Wehner, B., Corander, J., Hämeri, K., and Hussein, T.: Evaluation of a statistical forecast model for size-fractionated urban particle number concentrations using data from five European cities, J. Aerosol Sci., 66, 96-110, https://doi.org/10.1016/j.jaerosci.2013.08.012, 2013.

Molteni, U., Bianchi, F., Klein, F., El Haddad, I., Frege, C., Rossi, M. J., Dommen, J., and Baltensperger, U.: Formation of highly oxygenated organic molecules from aromatic compounds, Atmos. Chem. Phys., 18, 1909-1921, https://doi.org/10.5194/acp18-1909-2018, 2018.

Napari, I., Noppel, M., Vehkamäki, H., and Kulmala, M.: An improved model for ternary nucleation of sulfuric acid-ammonia-water, J. Chem. Phys., 116, 4221-4227, https://doi.org/10.1063/1.1450557, 2002. 
Nieminen, T., Lehtinen, K. E. J., and Kulmala, M.: Sub-10 nm particle growth by vapor condensation - effects of vapor molecule size and particle thermal speed, Atmos. Chem. Phys., 10, 97739779, https://doi.org/10.5194/acp-10-9773-2010, 2010.

Nieminen, T., Kerminen, V.-M., Petäjä, T., Aalto, P. P., Arshinov, M., Asmi, E., Baltensperger, U., Beddows, D. C. S., Beukes, J. P., Collins, D., Ding, A., Harrison, R. M., Henzing, B., Hooda, R., Hu, M., Hõrrak, U., Kivekäs, N., Komsaare, K., Krejci, R., Kristensson, A., Laakso, L., Laaksonen, A., Leaitch, W. R., Lihavainen, H., Mihalopoulos, N., Németh, Z., Nie, W., O'Dowd, C., Salma, I., Sellegri, K., Svenningsson, B., Swietlicki, E., Tunved, P., Ulevicius, V., Vakkari, V., Vana, M., Wiedensohler, A., Wu, Z., Virtanen, A., and Kulmala, M.: Global analysis of continental boundary layer new particle formation based on longterm measurements, Atmos. Chem. Phys., 18, 14737-14756, https://doi.org/10.5194/acp-18-14737-2018, 2018.

O’Dowd, C. D., Jimenez, J. L., Bahreini, R., Flagan, R. C., Seinfeld, J. H., Hämeri, K., Pirjola, L., Kulmala, M., Jennings, S. G. and Hoffmann, T.: Marine aerosol formation from biogenic iodine emissions, Nature, 417, 632-636, https://doi.org/10.1038/nature00775, 2002.

Olenius, T., Halonen, R., Kurtén, T., Henschel, H., Kupiainen-Määttä, O., Ortega, I. K., Jen, C. N., Vehkamäki, H., and Riipinen, I.: New particle formation from sulfuric acid amines: Comparison of monomethylamine, dimethylamine, and trimethylamine, J. Geophys. Res.-Atmos., 122, 7103-7118, https://doi.org/10.1002/2017JD026501, 2017.

Olin, M., Kuuluvainen, H., Aurela, M., Kalliokoski, J., Kuittinen, N., Isotalo, M., Timonen, H. J., Niemi, J. V., Rönkkö, T., and Dal Maso, M.: Traffic-originated nanocluster emission exceeds $\mathrm{H}_{2} \mathrm{SO}_{4}$-driven photochemical new particle formation in an urban area, Atmos. Chem. Phys., 20, 1-13, https://doi.org/10.5194/acp-20-1-2020, 2020.

Paasonen, P., Asmi, A., Petäjä, T., Kajos, M. K., Äijälä, M., Junninen, H., Holst, T., Abbatt, J. P. D., Arneth, A., Birmili, W., Van Der Gon, H. D., Hamed, A., Hoffer, A., Laakso, L., Laaksonen, A., Richard Leaitch, W., Plass-Dülmer, C., Pryor, S. C., Räisänen, P., Swietlicki, E., Wiedensohler, A., Worsnop, D. R., Kerminen, V. M., and Kulmala, M.: Warming-induced increase in aerosol number concentration likely to moderate climate change, Nat. Geosci., 6, 438-442, https://doi.org/10.1038/ngeo1800, 2013.

Park, M., Yum, S. S., and Kim, J. H.: Characteristics of submicron aerosol number size distribution and new particle formation events measured in Seoul, Korea, during 2004-2012, AsiaPac. J. Atmos. Sci., 51, 1-10, https://doi.org/10.1007/s13143014-0055-0, 2015.

Petäjä, T., Mauldin, III, R. L., Kosciuch, E., McGrath, J., Nieminen, T., Paasonen, P., Boy, M., Adamov, A., Kotiaho, T., and Kulmala, M.: Sulfuric acid and $\mathrm{OH}$ concentrations in a boreal forest site, Atmos. Chem. Phys., 9, 7435-7448, https://doi.org/10.5194/acp9-7435-2009, 2009.

Pikridas, M., Sciare, J., Freutel, F., Crumeyrolle, S., von der Weiden-Reinmüller, S.-L., Borbon, A., Schwarzenboeck, A., Merkel, M., Crippa, M., Kostenidou, E., Psichoudaki, M., Hildebrandt, L., Engelhart, G. J., Petäjä, T., Prévôt, A. S. H., Drewnick, F., Baltensperger, U., Wiedensohler, A., Kulmala, M., Beekmann, M., and Pandis, S. N.: In situ formation and spatial variability of particle number concentration in a European megacity, Atmos. Chem. Phys., 15, 10219-10237, https://doi.org/10.5194/acp-15-10219-2015, 2015.

Pillai, P., Khlystov, A., Walker, J., and Aneja, V.: Observation and analysis of particle nucleation at a forest site in southeastern US, Atmosphere-Basel, 4, 72-93, https://doi.org/10.3390/atmos4020072, 2013.

Poling, B. E., Prausnitz, J. M., and O'Connell, J. P.: The properties of gases and liquids, 5th edition, McGraw-Hill Education, New York, USA, 768 pp., 2001.

Politis, M., Pilinis, C., and Lekkas, T. D.: Ultrafine particles (UFP) and health effects, Dangerous, Like no other PM? Review and analysis, Global Nest J., 10, 439-452, 2008.

Quéléver, L. L. J., Kristensen, K., Normann Jensen, L., Rosati, B., Teiwes, R., Daellenbach, K. R., Peräkylä, O., Roldin, P., Bossi, R., Pedersen, H. B., Glasius, M., Bilde, M., and Ehn, M.: Effect of temperature on the formation of highly oxygenated organic molecules (HOMs) from alpha-pinene ozonolysis, Atmos. Chem. Phys., 19, 7609-7625, https://doi.org/10.5194/acp19-7609-2019, 2019.

Querol, X., Gangoiti, G., Mantilla, E., Alastuey, A., Minguillón, M. C., Amato, F., Reche, C., Viana, M., Moreno, T., Karanasiou, A., Rivas, I., Pérez, N., Ripoll, A., Brines, M., Ealo, M., Pandolfi, M., Lee, H.-K., Eun, H.-R., Park, Y.-H., Escudero, M., Beddows, D., Harrison, R. M., Bertrand, A., Marchand, N., Lyasota, A., Codina, B., Olid, M., Udina, M., Jiménez-Esteve, B., Soler, M. R., Alonso, L., Millán, M., and Ahn, K.-H.: Phenomenology of high-ozone episodes in NE Spain, Atmos. Chem. Phys., 17, 2817-2838, https://doi.org/10.5194/acp-17-2817-2017, 2017.

Riccobono, F., Schobesberger, S., Scott, C. E., Dommen, J., Ortega, I. K., Rondo, L., Almeida, J., Amorim, A., Bianchi, F., Breitenlechner, M., David, A., Downard, A., Dunne, E. M., Duplissy, J., Ehrhart, S., Flagan, R. C., Franchin, A., Hansel, A., Junninen, H., Kajos, M., Keskinen, H., Kupc, A., Makhmutov, V., Mathot, S., Nieminen, T., Onnela, A., Petäjä, T., Tsagkogeorgas, G., Vaattovaara, P., Viisanen, Y., Vrtala, A., and Wagner, P. E.: Oxidation Products of biogenic atmospheric particles, Science, 717, 717722, https://doi.org/10.1126/science.1243527, 2014.

Rimnácová, D., Ždímal, V., Schwarz, J., Smolík, J., and Rimnác, M.: Atmospheric aerosols in suburb of Prague: The dynamics of particle size distributions, Atmos. Res., 101, 539-552, https://doi.org/10.1016/j.atmosres.2010.10.024, 2011.

Rivas, I., Beddows, D. C. S., Amato, F., Green, D. C., Järvi, L., Hueglin, C., Reche, C., Timonen, H., Fuller, G. W., Niemi, J. V., Pérez, N., Aurela, M., Hopke, P. K., Alastuey, A., Kulmala, M., Harrison, R. M., Querol, X., and Kelly, F. J.: Source apportionment of particle number size distribution in urban background and traffic stations in four European cities, Environ. Int., 135, 105345, https://doi.org/10.1016/j.envint.2019.105345, 2020.

Rizzo, L. V., Artaxo, P., Karl, T., Guenther, A. B., and Greenberg, J.: Aerosol properties, in-canopy gradients, turbulent fluxes and VOC concentrations at a pristine forest site in Amazonia, Atmos. Environ., 44, 503-511, https://doi.org/10.1016/j.atmosenv.2009.11.002, 2010.

Rose, C., Zha, Q., Dada, L., Yan, C., Lehtipalo, K., Junninen, H., Mazon, S. B., Jokinen, T., Sarnela, N., Sipilä, M., Petäjä, T., Kerminen, V.-M., Bianchi, F., and Kulmala, M.: Observations of biogenic ion-induced cluster formation in the atmosphere, Sci. Adv., 4, eaar5218, https://doi.org/10.1126/sciadv.aar5218, 2018. 
Salma, I., Borsós, T., Weidinger, T., Aalto, P., Hussein, T., Dal Maso, M., and Kulmala, M.: Production, growth and properties of ultrafine atmospheric aerosol particles in an urban environment, Atmos. Chem. Phys., 11, 1339-1353, https://doi.org/10.5194/acp-11-1339-2011, 2011.

Schwartz, J., Dockery, D. W., and Neas, L. M.: Is Daily Mortality Associated Specifically with Fine Particles?, J. Air Waste Manage., 46, 927-939, https://doi.org/10.1080/10473289.1996.10467528, 1996.

Seinfeld, J. H. and Pandis, S. N.: Atmospheric Chemistry and Physics: From Air Pollution to Climate Change, 3rd edition, John Wiley \& Sons Inc, New Jersey, Canada, 2012.

Shen, X., Sun, J., Kivekäs, N., Kristensson, A., Zhang, X., Zhang, Y., Zhang, L., Fan, R., Qi, X., Ma, Q., and Zhou, H.: Spatial distribution and occurrence probability of regional new particle formation events in eastern China, Atmos. Chem. Phys., 18, 587599, https://doi.org/10.5194/acp-18-587-2018, 2018.

Shrivastava, M., Cappa, C. D., Fan, J., Goldstein, A. H., Guenther, A. B., Jimenez, J. L., Kuang, C., Laskin, A., Martin, S. T., Ng, N. L., Petaja, T., Pierce, J. R., Rasch, P. J., Roldin, P., Seinfeld, J. H., Shilling, J., Smith, J. N., Thornton, J. A., Volkamer, R., Wang, J., Worsnop, D. R., Zaveri, R. A., Zelenyuk, A., and Zhang, Q.: Recent advances in understanding secondary organic aerosol: Implications for global climate forcing, Rev. Geophys., 55, 509559, https://doi.org/10.1002/2016RG000540, 2017.

Siakavaras, D., Samara, C., Petrakakis, M., and Biskos, G.: Nucleation events at a coastal city during the warm period: Kerbside versus urban background measurements, Atmos. Environ., 140, 60-68, https://doi.org/10.1016/j.atmosenv.2016.05.054, 2016.

Sipila, M., Berndt, T., Petaja, T., Brus, D., Vanhanen, J., Stratmann, F., Patokoski, J., Mauldin III, R. L., Hyvarinen, A. P., Lihavainen, H., and Kulmala, M.: The Role of Sulfuric Acid in Atmospheric Nucleation, Science, 327, 1243-1246, https://doi.org/10.1126/science.1180315, 2010.

Spracklen, D. V., Carslaw, K. S., Merikanto, J., Mann, G. W., Reddington, C. L., Pickering, S., Ogren, J. A., Andrews, E., Baltensperger, U., Weingartner, E., Boy, M., Kulmala, M., Laakso, L., Lihavainen, H., Kivekäs, N., Komppula, M., Mihalopoulos, N., Kouvarakis, G., Jennings, S. G., O'Dowd, C., Birmili, W., Wiedensohler, A., Weller, R., Gras, J., Laj, P., Sellegri, K., Bonn, B., Krejci, R., Laaksonen, A., Hamed, A., Minikin, A., Harrison, R. M., Talbot, R., and Sun, J.: Explaining global surface aerosol number concentrations in terms of primary emissions and particle formation, Atmos. Chem. Phys., 10, 4775-4793, https://doi.org/10.5194/acp-10-4775-2010, 2010.

Stolzenburg, D., Fischer, L., Vogel, A. L., Heinritzi, M., Schervish, M. and Simon, M., Wagner, A. C., Dada, L., Ahonen, L. R., Amorim, A., Baccarini, A., Bauer, P. S., Baumgartner, B., Bergen, A., Bianchi, F., Breitenlechner, M., Brilke, S., Buenorstro Mazon, S., Chen, D., Dias, A., Draper, D. C., Duplissy, J., El Haddad, I., Finkenzeller, H., Frege, C., Fuchs, C., Garmash, O., Gordon, H., He, X., Helm., J., Hofbauer, V., Hoyle, C. R., Kim, C., Kirkby, J., Kontkanen, J., Kürten, A., Lampilahti, J., Lawler, M., Lehtipalo, K., Leiminger, M., Mai, H., Mathot, S., Mentler, B., Molteni, U., Nie, W., Nieminen, T., Nowak, J. B., Ojdanic, A., Onnela, A., Passananti, M., Petäjä, T., Quéléver, L. L. J., Rissanen, M. P., Sarnela, N., Schallhart, S., Tauber, C., Tome, A., Wagner, R., Wang, M., Weitz, L., Wimmer, D., Xiao, M., Yan, C., Ye, P., Zha, Q., Baltensperger, U., Curtius, J.,
Dommen, J., Flagan, R. C., Kulmala, M., Smith, J. N., Worsnop, D. R., Hansel, A., Donahue, N. M., and Winkler, P. M.: Rapid growth of organic aerosol nanoparticles over a wide tropospheric temperature range, P. Natl. Acad. Sci. USA, 115, 9122-9127, https://doi.org/10.1073/pnas.1807604115, 2018.

Stolzenburg, D., Simon, M., Ranjithkumar, A., Kürten, A., Lehtipalo, K., Gordon, H., Ehrhart, S., Finkenzeller, H., Pichelstorfer, L., Nieminen, T., He, X.-C., Brilke, S., Xiao, M., Amorim, A., Baalbaki, R., Baccarini, A., Beck, L., Bräkling, S., Caudillo Murillo, L., Chen, D., Chu, B., Dada, L., Dias, A., Dommen, J., Duplissy, J., El Haddad, I., Fischer, L., Gonzalez Carracedo, L., Heinritzi, M., Kim, C., Koenig, T. K., Kong, W., Lamkaddam, H., Lee, C. P., Leiminger, M., Li, Z., Makhmutov, V., Manninen, H. E., Marie, G., Marten, R., Müller, T., Nie, W., Partoll, E., Petäjä, T., Pfeifer, J., Philippov, M., Rissanen, M. P., Rörup, B., Schobesberger, S., Schuchmann, S., Shen, J., Sipilä, M., Steiner, G., Stozhkov, Y., Tauber, C., Tham, Y. J., Tomé, A., VazquezPufleau, M., Wagner, A. C., Wang, M., Wang, Y., Weber, S. K., Wimmer, D., Wlasits, P. J., Wu, Y., Ye, Q., Zauner-Wieczorek, M., Baltensperger, U., Carslaw, K. S., Curtius, J., Donahue, N. M., Flagan, R. C., Hansel, A., Kulmala, M., Lelieveld, J., Volkamer, R., Kirkby, J., and Winkler, P. M.: Enhanced growth rate of atmospheric particles from sulfuric acid, Atmos. Chem. Phys., 20, 7359-7372, https://doi.org/10.5194/acp20-7359-2020, 2020.

Tröstl, J., Chuang, W. K., Gordon, H., Heinritzi, M., Yan, C., Molteni, U., Ahlm, L., Frege, C., Bianchi, F., Wagner, R., Simon, M., Lehtipalo, K., Williamson, C., Craven, J. S., Duplissy, J., Adamov, A., Almeida, J., Bernhammer, A. K., Breitenlechner, M., Brilke, S., Dias, A., Ehrhart, S., Flagan, R. C., Franchin, A., Fuchs, C., Guida, R., Gysel, M., Hansel, A., Hoyle, C. R., Jokinen, T., Junninen, H., Kangasluoma, J., Keskinen, H., Kim, J., Krapf, M., Kürten, A., Laaksonen, A., Lawler, M., Leiminger, M., Mathot, S., Möhler, O., Nieminen, T., Onnela, A., Petäjä, T., Piel, F. M., Miettinen, P., Rissanen, M. P., Rondo, L., Sarnela, N., Schobesberger, S., Sengupta, K., Sipilä, M., Smith, J. N., Steiner, G., Tomè, A., Virtanen, A., Wagner, A. C., Weingartner, E., Wimmer, D., Winkler, P. M., Ye, P., Carslaw, K. S., Curtius, J., Dommen, J., Kirkby, J., Kulmala, M., Riipinen, I., Worsnop, D. R., Donahue, N. M., and Baltensperger, U.: The role of low-volatility organic compounds in initial particle growth in the atmosphere, Nature, 533, 527-531, https://doi.org/10.1038/nature18271, 2016.

von Bismarck-Osten, C. and Weber, S.: A uniform classification of aerosol signature size distributions based on regression-guided and observational cluster analysis, Atmos. Environ., 89, 346357, https://doi.org/10.1016/j.atmosenv.2014.02.050, 2014.

von Bismarck-Osten, C. Birmili, W., Ketzel, M., Massling, A., Petäjä, T., and Weber, S.: Characterization of parameters influencing the spatio-temporal variability of urban particle number size distributions in four European cities, Atmos. Environ., 77, 415-429, https://doi.org/10.1016/j.atmosenv.2013.05.029, 2013. von Bismarck-Osten, C., Birmili, W., Ketzel, M., and Weber, S.: Statistical modelling of aerosol particle number size distributions in urban and rural environments - A multi-site study, Urban Climate, 11, 51-66, https://doi.org/10.1016/j.uclim.2014.11.004, 2015.

Vratolis, S., Gini, M. I., Bezantakos, S., Stavroulas, I., Kalivitis, N., Kostenidou, E., Louvaris, E., Siakavaras, D., Biskos, G., Mi- 
halopoulos, N., Pandis, S. N. N., Pilinis, C., Papayannis, A., and Eleftheriadis, K.: Particle number size distribution statistics at City-Centre Urban Background, urban background, and remote stations in Greece during summer, Atmos. Environ., 213, 711726, https://doi.org/10.1016/j.atmosenv.2019.05.064, 2019.

Wagner, P. and Kuttler, W.: Biogenic and anthropogenic isoprene in the near-surface urban atmosphere - A case study in Essen, Germany, Sci. Total Environ., 475, 104-115, https://doi.org/10.1016/j.scitotenv.2013.12.026, 2014.

Wang, F., Ketzel, M., Ellermann, T., Wåhlin, P., Jensen, S. S., Fang, D., and Massling, A.: Particle number, particle mass and $\mathrm{NO}_{x}$ emission factors at a highway and an urban street in Copenhagen, Atmos. Chem. Phys., 10, 2745-2764, https://doi.org/10.5194/acp-10-2745-2010, 2010.

Wang, M., Kong, W., Marten, R., He, X. C., Chen, D., Pfeifer, J., Heitto, A., Kontkanen, J., Dada, L., Kürten, A., Yli-Juuti, T., Manninen, H. E., Amanatidis, S., Amorim, A., Baalbaki, R., Baccarini, A., Bell, D. M., Bertozzi, B., Bräkling, S., Brilke, S., Murillo, L. C., Chiu, R., Chu, B., De Menezes, L. P., Duplissy, J., Finkenzeller, H., Carracedo, L. G., Granzin, M., Guida, R., Hansel, A., Hofbauer, V., Krechmer, J., Lehtipalo, K., Lamkaddam, H., Lampimäki, M., Lee, C. P., Makhmutov, V., Marie, G., Mathot, S., Mauldin, R. L., Mentler, B., Müller, T., Onnela, A., Partoll, E., Petäjä, T., Philippov, M., Pospisilova, V., Ranjithkumar, A., Rissanen, M., Rörup, B., Scholz, W., Shen, J., Simon, M., Sipilä, M., Steiner, G., Stolzenburg, D., Tham, Y. J., Tomé, A., Wagner, A. C., Wang, D. S., Wang, Y., Weber, S. K., Winkler, P. M., Wlasits, P. J., Wu, Y., Xiao, M., Ye, Q., ZaunerWieczorek, M., Zhou, X., Volkamer, R., Riipinen, I., Dommen, J., Curtius, J., Baltensperger, U., Kulmala, M., Worsnop, D. R., Kirkby, J., Seinfeld, J. H., El-Haddad, I., Flagan, R. C., and Donahue, N. M.: Rapid growth of new atmospheric particles by nitric acid and ammonia condensation, Nature, 581, 184-189, https://doi.org/10.1038/s41586-020-2270-4, 2020.

Wang, S., Wu, R., Berndt, T., Ehn, M., and Wang, L.: Formation of Highly Oxidized Radicals and Multifunctional Products from the Atmospheric Oxidation of Alkylbenzenes, Environ. Sci. Technol., 51, 8442-8449, https://doi.org/10.1021/acs.est.7b02374, 2017.

Wang, Z., Wu, Z., Yue, D., Shang, D., Guo, S., Sun, J., Ding, A., Wang, L., Jiang, J., Guo, H., Gao, J., Cheung, H. C., Morawska, L., Keywood, M., and Hu, M.: New particle formation in China: Current knowledge and further directions, Sci. Total Environ., 577, 258-266, https://doi.org/10.1016/j.scitotenv.2016.10.177, 2017.

Weber, R. J., McMurry, P. H., Eisele, F. L., and Tanner, D. J.: Measurement of expected nucleation precursor species and 3$500 \mathrm{~nm}$ diameter particles at Mauna Loa Observatory, Hawaii, J. Atmos. Sci., 52, 2242-2257, https://doi.org/10.1175/15200469(1995)052<2242:MOENPS>2.0.CO;2, 1995.

Wehner, B., Siebert, H., Stratmann, F., Tuch, T., Wiedensohler, A., Petäjä, T., Dal Maso, M., and Kulmala, M.: Horizontal homogeneity and vertical extent of new particle formation events, Tellus B, 59, 362-371, https://doi.org/10.1111/j.16000889.2007.00260.x, 2007.
Wiedensohler, A., Ma, N., Birmili, W., Heintzenberg, J., Ditas, F., Andreae, M. O., and Panov, A.: Infrequent new particle formation over the remote boreal forest of Siberia, Atmos. Environ., 200, 167-169, https://doi.org/10.1016/j.atmosenv.2018.12.013, 2019.

Wonaschütz, A., Demattio, A., Wagner, R., Burkart, J., Zíková, N., Vodička, P., Ludwig, W., Steiner, G., Schwarz, J., and Hitzenberger, R.: Seasonality of new particle formation in Vienna, Austria - Influence of air mass origin and aerosol chemical composition, Atmos. Environ., 118, 118-126, https://doi.org/10.1016/j.atmosenv.2015.07.035, 2015.

Woo, K. S., Chen, D. R., Pui, D. Y. H. H., and McMurry, P. H.: Measurement of Atlanta aerosol size distributions: Observations of lutrafine particle events, Aerosol Sci. Tech., 34, 75-87, https://doi.org/10.1080/02786820120056, 2001.

Yamada, H.: Contribution of evaporative emissions from gasoline vehicles toward total VOC emissions in Japan, Sci. Total Environ., 449, 143-149, https://doi.org/10.1016/j.scitotenv.2013.01.045, 2013.

Yan, C., Dada, L., Rose, C., Jokinen, T., Nie, W., Schobesberger, S., Junninen, H., Lehtipalo, K., Sarnela, N., Makkonen, U., Garmash, O., Wang, Y., Zha, Q., Paasonen, P., Bianchi, F., Sipilä, M., Ehn, M., Petäjä, T., Kerminen, V.-M., Worsnop, D. R., and Kulmala, M.: The role of $\mathrm{H}_{2} \mathrm{SO}_{4}-\mathrm{NH}_{3}$ anion clusters in ion-induced aerosol nucleation mechanisms in the boreal forest, Atmos. Chem. Phys., 18, 13231-13243, https://doi.org/10.5194/acp-1813231-2018, 2018.

Yan, C., Nie, W., Vogel, A. L., Dada, L., Lehtipalo, K., Stolzenburg, D., and Wagner, R.: Size-dependent influence of $\mathrm{NO}_{x}$ on the growth rates of organic aerosol particles, Sci. Adv., 6, 1-10, 2020.

Yao, L., Garmash, O., Bianchi, F., Zheng, J., Yan, C., Kontkanen, J., Junninen, H., Mazon, S. B., Ehn, M., Paasonen, P., Sipilä, M., Wang, M., Wang, X., Xiao, S., Chen, H., Lu, Y., Zhang, B., Wang, D., Fu, Q., Geng, F., Li, L., Wang, H., Qiao, L., Yang, X., Chen, J., Kerminen, V.-M., Petäjä, T., Worsnop, D. R., Kulmala, M., and Wang, L.: Atmospheric new particle formation from sulfuric acid and amines in a Chinese megacity, Science, 361, 278281, https://doi.org/10.1126/science.aao4839, 2018.

Ye, J., Abbatt, J. P. D., and Chan, A. W. H.: Novel pathway of $\mathrm{SO}_{2}$ oxidation in the atmosphere: reactions with monoterpene ozonolysis intermediates and secondary organic aerosol, Atmos. Chem. Phys., 18, 5549-5565, https://doi.org/10.5194/acp18-5549-2018, 2018.

Yli-Juuti, T., Mohr, C., and Riipinen, I.: Open questions on atmospheric nanoparticle growth, Commun. Chem., 3, 106, https://doi.org/10.1038/s42004-020-00339-4, 2020.

Zhang, R., Khalizov, A., Wang, L., Hu, M., and Xu, W.: Nucleation and growth of nanoparticles in the atmosphere, Chem. Rev., 112, 1957-2011, https://doi.org/10.1021/cr2001756, 2012. 